\title{
Aldol condensation of biomass-derived levulinic acid and furfural over acid zeolites
}

Jennifer Cueto ${ }^{1}$, Valeria Korobka ${ }^{2}$, Laura Faba ${ }^{1}$, Eva Díaz ${ }^{1}$, Salvador Ordóñez ${ }^{1 *}$

${ }^{1}$ Catalysis, Reactors and Control Research Group (CRC), Dept. of Chemical and Environmental Engineering, University of Oviedo, Oviedo 33006, Spain

${ }^{2}$ Department of Chemical and Process Engineering, University of Strathclyde, 75 Montrose Street, Glasgow G11XJ, Scotland, UK

*e-mail: sordonez@uniovi.es, Tel: +34 985103 437; Fax: + 34985103434

ABSTRACT. Acid-catalysed cross condensation between furfural and levulinic acid is presented in this work as an alternative for manufacturing C10 chemicals from renewable platform molecules. First experiments conclude that the neutralization of the carboxylic group of levulinic acid is required to prevent side reactions such as cyclization. Two C10 products are obtained, suggesting two parallel reaction mechanisms. Different reaction conditions (temperatures and initial reactants ratios) have been tested, and results were used to propose a kinetic model. The role of the properties of different catalysts is also studied, analysing the activity, selectivity and acidities of different zeolites structures (ZSM-5, MOR, BETA), observing a clear correspondence between medium-strength acidity and the production of the most stable product. A high selectivity to this compound is required to reduce the permanent adsorption and oligomerization of the starting materials, the main deactivation cause identified in this process. Thus, the best results were obtained with ZSM-5 (23) at $423 \mathrm{~K}$ and a LA:FFL ratio 2:1, with $50 \%$ of products yield and almost negligible relevance of side reactions (carbon balance after 24 h: $91 \%)$, observing a good behaviour after three reusability cycles without any regeneration.

KEYWORDS: Biomass upgrading, sodium levulinate, acid catalysis, ZSM-5, C-C coupling, biorefinery. 


\section{INTRODUCTION}

In the new strategies for replacing petrochemicals by renewable carbon-based chemicals, the difficult for selectively preparing chemicals with carbon chains of more than six carbon atoms is still a huge challenge, since most of the biobased platform molecules have six or less carbon atoms. ${ }^{1,2}$ Aldol condensation reactions are considered among the most interesting reactions to produce $\mathrm{C}-\mathrm{C}$ bonds between these bio-platform molecules. ${ }^{3-8}$ Despite the existence of other alternatives (such as Claisen, Knoevenagel, Guerbet condensations, or biological processes, among others), ${ }^{9-12}$ aldolization stands out because of the mild reaction conditions used (typically ambient pressure and temperatures below $450 \mathrm{~K}$ ), and the huge amount of potential raw materials directly obtained from biomass (furfural -FFL-, 5-HMF, acetone, cyclopentanone, etc.), with two alternative coupling mechanisms known (via enol or enolate intermediate, as function of using acid or basic catalysts, respectively). ${ }^{13-15}$

Levulinic acid (LA) is a very interesting bio-platform molecule. ${ }^{16,17}$ Several alternative production routes are reported in the literature, the most promising one being the Biofine process and its production via furfuryl alcohol, with final yields higher than $70 \%$ using commercial zeolites. ${ }^{18,19}$ LA is proposed as starting compound of many different processes, most of them involving its internal cyclization and the subsequent hydrogenation to $\mathrm{Y}$ valerolactone, ${ }^{20-23}$ a reaction in principle known since the 1930 's. ${ }^{24}$ Typically, the carboxylic group of LA plays a key role in the mechanism, other routes involving the activity of the 
carbonyl group being scarcely investigated, as is the case of aldol condensation. This reaction would allow obtaining a family of C10 compounds with promising uses as fuel additives, surfactants, lubricants or pharmaceutical precursors. ${ }^{25,26}$

LA self-condensation has been proposed considering basic and acid materials (mixed oxides, Amberlyst-15, etc.), concluding that results are strongly conditioned by the competition between the activity of the carbonyl group and the carboxyl one. ${ }^{25-27}$ Thus, more than $30 \%$ of angelica lactone (C5) is produced at $323 \mathrm{~K}$, with only $40 \% \mathrm{LA}$ conversion. The yield of this compound increases with the temperature, being the main limitation of this procedure..$^{25}$

The predominance of cyclization suggests that LA self-condensation is not fast enough to prevail over this undesired side reaction. The cross-condensation approach, involving a more active molecule, is the natural way to continue these studies, with FFL or 5 HMF being good alternatives, successfully tested in similar reactions. ${ }^{13,28,29}$ Both cyclic aldehydes are obtained by the hydrolysis and dehydration of sugar polymers. ${ }^{30,31}$ Thus, the co-production of both reactants is an additional advantage, reducing the purification steps required. However, LA is obtained by the rehydration of 5-HMF under acidic conditions, so the use of this molecule is not suggested, to avoid interactions related to the decomposition of $5 \mathrm{HMF}$ into LA. ${ }^{32,33} \mathrm{FFL}$ has been previously proposed as a co-reactant in most of the aldol condensation processes used, obtaining very promising results with different platform molecules. ${ }^{34-36}$ Despite its well-known activity, there are only two papers considering LA and FFL condensation. In the first one, $\mathrm{NaOH}$ is proposed as catalyst, giving $95 \%$ yield after $48 \mathrm{~h}$ reaction time at $273 \mathrm{~K} .{ }^{37}$ Despite the undoubted relevance of this study as a first approach to the reaction mechanism, the use of 
homogeneous catalysis is against of the main Principles of Green Chemistry. Concerning the heterogeneous catalysts, different materials were tested in a preliminary screening in a second study. ${ }^{38}$ In general, FFL conversions lower than $40 \%$ were reported, except in the cases where overall selectivities are far from $100 \%$, suggesting the presence of undesired reactions. In this context, a deep study is required, since the reaction conditions were not optimized, and no references to side reactions, carbon balances or deactivation were included.

The aim of this work is to present the results obtained in the study of FFL and LA crosscondensation using different acid zeolites (widely available acid catalysts). Reactions conditions were optimized, in terms of temperature and initial reactants ratio. The role of acidity as well as structural properties of zeolitic catalysts was also studied. The experimental data were used to propose a kinetic model. Reusability tests were also carried out with reaction conditions previously optimized.

\section{EXPERIMENTAL SECTION}

\section{Catalysts and characterization}

$\mathrm{NH}_{4}-\mathrm{ZSM}-5$ (CBV 2314), $\mathrm{NH}_{4}-\mathrm{ZSM}-5$ (CBV 8014), $\mathrm{NH}_{4}-\mathrm{MOR}$ (CBV 21A) and $\mathrm{NH}_{4}-\mathrm{BETA}$ (CP 814E) were provided by Zeolyst International. The framework $\mathrm{SiO}_{2} / \mathrm{Al}_{2} \mathrm{O}_{3}$ ratios were $23,80,20$ and 25 , respectively. These materials have been activated at $823 \mathrm{~K}$ for $12 \mathrm{~h}$ to transform $\mathrm{NH}_{4}$ functional groups into $\mathrm{H}$-forms. 
Acidic properties of these catalysts have been evaluated by two different techniques: titration with $\mathrm{NaOH}$ and temperature programmed desorption of $\mathrm{NH}_{3}\left(\mathrm{NH}_{3}-\mathrm{TPD}\right)$ using a Micromeritics TPD/TPR 2900. With respect to the first one, $25 \mathrm{mg}$ of each catalyst were introduced into $50 \mathrm{ml}$ of $0.1 \mathrm{M}$ solution of $\mathrm{NaCl}$, acidifying the mixture previously with $0.5 \mathrm{ml}$ of $0.1 \mathrm{M} \mathrm{HCl}$. After $3 \mathrm{~h}$ of stirring at room temperature, different volumes of $0.1 \mathrm{M} \mathrm{NaOH}$ were added by measuring with each addition the $\mathrm{pH}$ until neutral $\mathrm{pH}$ was obtained. In addition, a titration of a blank was performed in order to calculate the total acidity by subtracting the volume of $\mathrm{NaOH}$ consumed with each catalyst from the corresponding one of the blank. Thus, the meq $\mathrm{H}^{+} / \mathrm{g}$ were obtained for each material analysed. Concerning the TPD analyses, $\mathrm{NH}_{3}$ signal evolution was measured with Pfeiffer Vacuum Omnistar Quadrupole Mass Spectrometer. 20 mg of sample were employed in each analysis. In order to remove any compound physisorbed on the surface, samples were pretreated before each analysis, by heating to $383 \mathrm{~K}$ in helium flow $\left(20 \mathrm{ml} \cdot \mathrm{min}^{-1}\right)$ for $1 \mathrm{~h}$. Subsequently, the catalytic surface was saturated in $\mathrm{NH}_{3}$ flow $\left(2.5 \% \mathrm{NH}_{3} / \mathrm{He}, 20 \mathrm{ml} \cdot \mathrm{min}^{-}\right.$ $\left.{ }^{1}\right)$ at room temperature for $15 \mathrm{~min}$. An intermediate stage of stabilization under helium flow was required to prevent signals related to oversaturation. Then, the desorption of physisorbed $\mathrm{NH}_{3}$ was monitored, being flushed with helium $\left(20 \mathrm{ml} \cdot \mathrm{min}^{-1}\right)$ from room temperature to $823 \mathrm{~K}$ with a temperature rate of $5 \mathrm{~K} \cdot \mathrm{min}^{-1}$. Morphological-properties of the zeolites (surface area, pore diameter and micro- and total pore volumes) were determined by nitrogen physisorption at $77 \mathrm{~K}$ using an ASAP 2020 (Micromeritics) static volumetric apparatus after the a degasification step at $383 \mathrm{~K}$ during $6 \mathrm{~h}$. 


\section{Catalytic performance}

LA and FFL aldol condensation was carried out in a $0.5 \mathrm{~L}$ stirred batch autoclave reactor equipped with a PID temperature controller and a backpressure regulator (Autoclave Engineers EZE Seal). Firstly, $100 \mathrm{ml}$ of an aqueous solution of LA (2 M, Sigma Aldrich, 99\%) was neutralized with a 0.1 mol of $\mathrm{Na}_{2} \mathrm{CO}_{3}$ (Panreac, $99 \%$ ) obtaining the corresponding salt (sodium levulinate, labelled as NaLe). The reactor was loaded with $0.25 \mathrm{~L}$ of an aqueous solution of FFL (Sigma Aldrich, $99 \%$ ) and NaLe solution (5 \% wt. of reactants) and $1.8 \mathrm{~g}$ of zeolite $(50-80 \mu \mathrm{m})$. Different reactants ratios were considered, the specific values being detailed in each corresponding reaction. Considering that a blank experiment allows discounting the self-catalytic reaction, the feed was pre-heated to $343 \mathrm{~K}$ before the catalyst was introduced. Thus, the starting point is better defined, minimizing mistakes related to reaction evolution before reaching the desired temperature. The analysis of reaction mixture at initial time discounts any decomposition or undesired reaction of any reactants. Different reaction temperatures were studied, from 323 to $448 \mathrm{~K}$, the particular value being indicated in each analysis. Before starting the reaction, the reactor is purged with nitrogen, and the reaction is carried out under 15 bar of $\mathrm{N}_{2}$ with a stirring of $1000 \mathrm{rpm}$ for $24 \mathrm{~h}$. Operation pressure was selected for both ensuring the absence of water or furfural evaporation at any working conditions, since their boiling points ( $373 \mathrm{~K}$ and $434 \mathrm{~K}$, respectively) are lower than the maximum temperature tested; and guaranteeing an excess enough to take the samples from the sampling point under safety conditions, (a overpressure is required to pump the liquid) without producing a relevant decrease in the reactor pressure that can favors the evaporation of any compound after taking several samples. 
Samples were taken from the reactor to obtain the evolution of reactants and products concentration during the reaction. A filter was placed in the sample port in order to prevent the extraction of catalyst and possible solids, which had formed during the reaction. The samples were extracted with ethyl acetate (volume ratio of 1:1) and acidified with $\mathrm{HCl}$ (2:1 volume ratio aqueous phase: solution $1 \mathrm{M}$ of $\mathrm{HCl}$ ). The organic phase was analysed by GC in a Shimadzu GC2010 equipped with a FID detector, using a 30 m long CP-Sil 8 CB capillary column. Peak assignment was carried out by GC-MS in a Shimadzu GC/MS QP 2010 Plus Instrument, using a $30 \mathrm{~m}$ long TRB-5MS capillary column. The calibrations of LA and FFL were performed using authentic samples, whereas the calibration of the products (labelled as $\delta-C 10$ and $\beta-C 10)$ was done using the relative carbon concept proposed by Scanlon and Willis ${ }^{39}$ since they are not commercially available. Catalysis results were described in terms of conversion, atomic yield and carbon balance closure, being calculated as follows (Equations 1-3):

$$
\begin{gathered}
\text { Conversion }(\%)=100 \cdot \frac{\left(\text { reactant }_{t=0}-\text { reactant }_{t}\right)}{\text { reactant }_{t=0}} \\
\Psi_{C 10}(\%)=100 \cdot \frac{10 \cdot([\delta-\mathrm{C} 10]+[\beta-\mathrm{C} 10])_{t}}{(5 \cdot[L A]+5[F F L])_{t=0}} \\
\text { C.B. }(\%)=100 \cdot \frac{10 \cdot([\delta-\mathrm{C} 10]+[\beta-\mathrm{C} 10])_{t}+5 \cdot([L A]+[F F L])_{t}}{(5 \cdot[L A]+5 \cdot[F F L])_{t=0}}
\end{gathered}
$$

The spent catalysts were recovered by filtration, washed and dried overnight at $373 \mathrm{~K}$ before their analysis by different techniques. TPD analyses were performed to determine possible adsorption of different compounds on the catalyst's surface. These experiments were carried out using a Micromeritics TPD/TPR 2900 with a helium flow of $20 \mathrm{ml} \cdot \mathrm{min}^{-1}$ increasing the temperature up to $1273 \mathrm{~K}$ with a $5 \mathrm{~K} \cdot \mathrm{min}^{-1}$ rate. Initial TPDs of fresh catalysts were done to 
ensure that materials were stable to the maximum temperature analyzed. The evolution of different signals was measured with a Pfeiffer Vacuum Omnistar Quadrupole Mass Spectrometer. Experimental results were compared to the $\mathrm{m} / \mathrm{z}$ spectra of all the compounds involved, according to data compiled by NIST Mass Spec Data Centre for reactants and the experimental spectrums obtained by GC-MS technique for products ( $\delta-C 10$ and $\beta-C 10)$ : FFL $(\mathrm{m} / \mathrm{z}=29,38,39,67,95,96) ; \mathrm{LA}(\mathrm{m} / \mathrm{z}=43,56,73,101,116) ; \delta-C 10(65,93,121,148,177,194) ;$ and $\beta-C 10(77,78,79,105,108,150,194)$. Considering that the mass spectrometer is more sensitive for small $\mathrm{m} / \mathrm{z}$ values, 39, 43, 65 and 77 were chosen as the signals for the analyses for $\mathrm{FFL}, \mathrm{LA}, \delta-\mathrm{C} 10$ and $\beta-C 10$, respectively. Using the same equipment and procedure, but introducing an oxidizing atmosphere $\left(20 \mathrm{ml} \cdot \mathrm{min}^{-1}\right.$ of $2 \% \mathrm{O}_{2}$ in He), TPO (temperatureprogrammed oxidation) tests were carried out to compare different carbonaceous deposits on catalyst surfaces by monitoring the $\mathrm{CO}_{2}$ release.

\section{RESULTS AND DISCUSION}

\section{Reaction mechanism and kinetic model}

Considering our previous results with LA self-condensation, ${ }^{25}$ a $\mathrm{Mg}$-Zr mixed oxide catalyst was proposed as a first approach to LA and FFL cross-condensation. The reaction was carried out at $323 \mathrm{~K}$, with equimolar initial reactants ratio and a catalytic loading of $0.5 \mathrm{~g}$. No C10 compounds were detected but a large amount of angelica lactone was formed (final yield of $12 \%$ with LA conversion of $21 \%$ after $24 \mathrm{~h}$ ). This reaction has been previously reported in the literature. ${ }^{40}$ This result suggests that internal LA cyclization prevails over cross-condensation, i.e., the 
homogeneous catalysis by the low $\mathrm{pH}$ of a levulinic acid solution $(\mathrm{pH}=2.5)$ prevails over the activity of the solid catalyst. According to this, the carboxylic acid functional group must be neutralized to prevent this undesired process. In addition, the carbon balance strongly decreases ( $54 \%$ ) being explained by the strong interaction between FFL (and LA at a lesser extent) and medium strength basic-acid pairs of Mg-Zr mixed oxide. This interaction enhances the permanent adsorption, causing oligomerization to solids blocking the active sites.

Based on these conclusions, the basic catalysis is abandoned, focusing the study on the activity of acid catalysts, such as ZSM-5 zeolite. This material has been widely proposed as catalyst for different biomass upgrading reactions, ${ }^{41,42}$ highlighting its activity in reactions requiring strong acidity ${ }^{43,44}$ To prevent the competence between the carboxylic group of LA and the carbonyl one (promoting undesired reactions such as cyclization), a previous esterification with ethanol was considered, studying the reaction between ethyl levulinate (EL) and FFL. Ethanol is chosen due to its renewable origin and non-hazard character, trying to respect the Principles of Green Chemistry. No conversions are observed when working at $323 \mathrm{~K}$ and $0.5 \mathrm{~g}$ of ZSM 5. The reaction temperature was then increased up to $373 \mathrm{~K}$, but no $\mathrm{C} 10$ compounds are detected even at these conditions. By contrast, LA is obtained ( $7 \%$ as yield after $24 \mathrm{~h}$ ), suggesting that esterification is partially reversible at these conditions. This equilibrium has been previously proposed in the literature..$^{45}$ The negligible formation of angelica lactone is congruent with the previous result (higher concentrations of LA are required to obtain a quantifiable cyclization), and the more neutral pH of this configuration ( 3.5 vs. 2.5 when working with LA). To sum up, using EL as reactant, there is not good balance between the severity of the required reaction 
conditions and the stability of the resulting ester. Thus, this was also abandoned for further investigations.

In a third approach, the neutralization with an inorganic salt $\left(\mathrm{Na}_{2} \mathrm{CO}_{3}\right)$ is proposed, in order to effect the desired reaction between sodium levulinate (NaLe) and FFL. In this approach, the use of the salt requires a subsequent acidification of products obtained, using $\mathrm{HCl}$ (details given in the Experimental section). Despite this procedure may challenge the sustainability of this process, both reactants were chosen considering the required neutralization with the lowest hazard level of wastes generated $(\mathrm{NaCl})$.

The evolution of reactants conversion and products yields, as well as the carbon balance closure is shown in Figure 1. The reaction is carried out at $373 \mathrm{~K}$ and using $1.8 \mathrm{~g}$ of ZSM 5 (23) and equimolar ratio of both reactants. Negligible reactant conversions were observed at lower temperatures. 


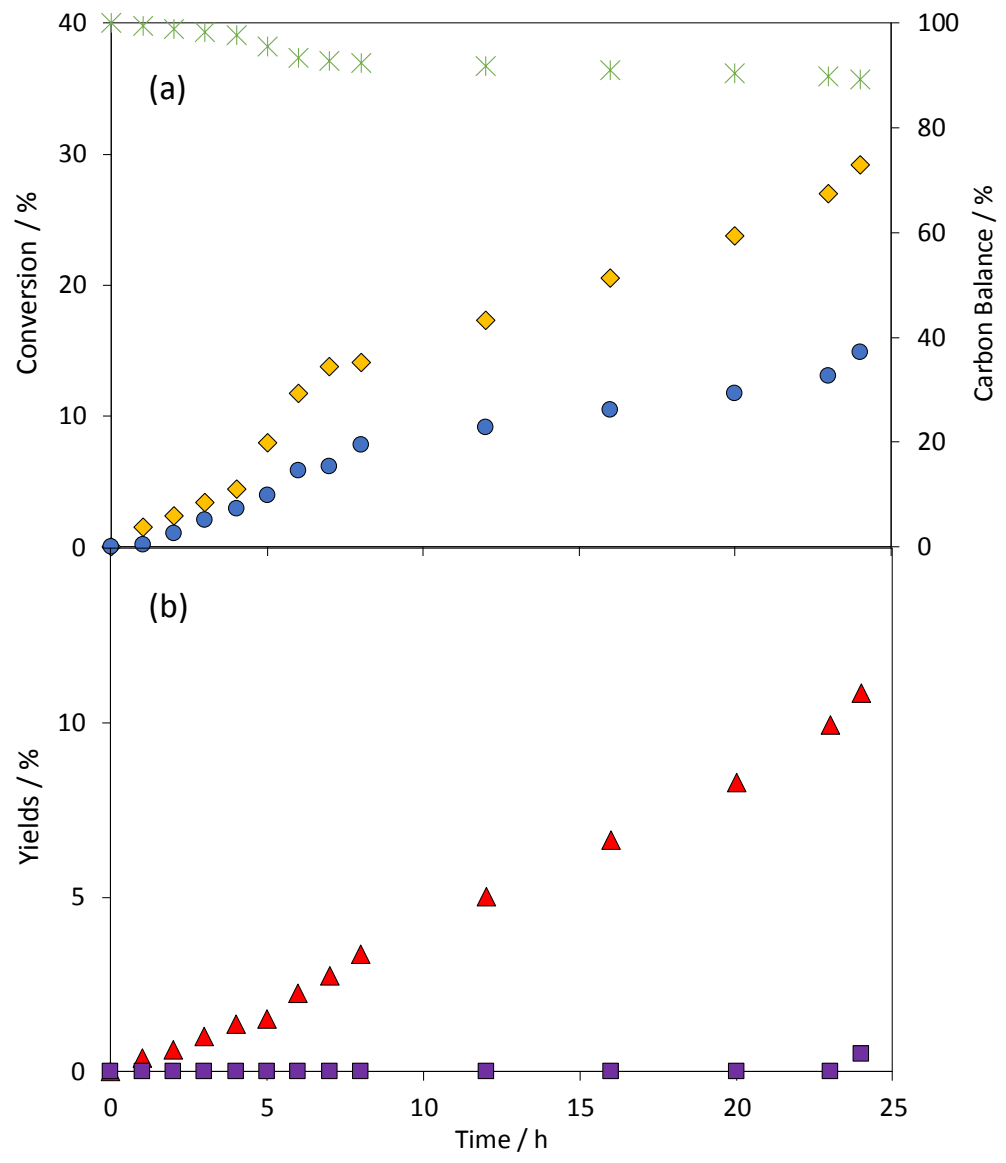

Figure 1. Temporal evolution of (a) reactants conversions and (b) product yields. Results corresponding to NaLe-FFL aldol condensation at $373 \mathrm{~K}$, using $1.8 \mathrm{~g}$ of ZSM-5 (23) and equimolar reactant ratio. Symbols: $(\bullet)$ NaLe; $(\bullet) \mathrm{FFL}$; $(*)$ carbon balance; $(\boldsymbol{\Delta}) \beta-C 10 ;(\boldsymbol{\square}) \delta$ C10.

The highest FFL conversion observed (29.2\% after 24 h) in comparison to the $14.9 \%$ obtained with NaLe (this conversion could directly referred to the levulinic acid one since the GC analysis is carried out once it was acidified with $\mathrm{HCl}$, the LA being the compound quantified), as well as the lack of correspondence with the products total yield (11.3\%) suggest the permanent adsorption or even oligomerization of FFL on the catalyst's surface. This hypothesis is congruent with the carbon balance, showing a progressive decrease to $89.2 \%$ after $24 \mathrm{~h}$. As to the products analysis (once, four main peaks were detected. According to the $\mathrm{m} / \mathrm{z}$ spectra obtained 
by GC-MS (shown in Figure S1), they can be grouped in two different C10 products: a branched compound labelled as $\beta-\mathrm{C} 10$ (main product, with a final yield of $10.8 \%$ ); and a linear one, $\delta$-C10 (0.5\% final yield). Each compound has two isomers (cis and trans), considered together in the analysis. Globally, the reaction mechanism suggested by the experimental results is summarized in Scheme 1.

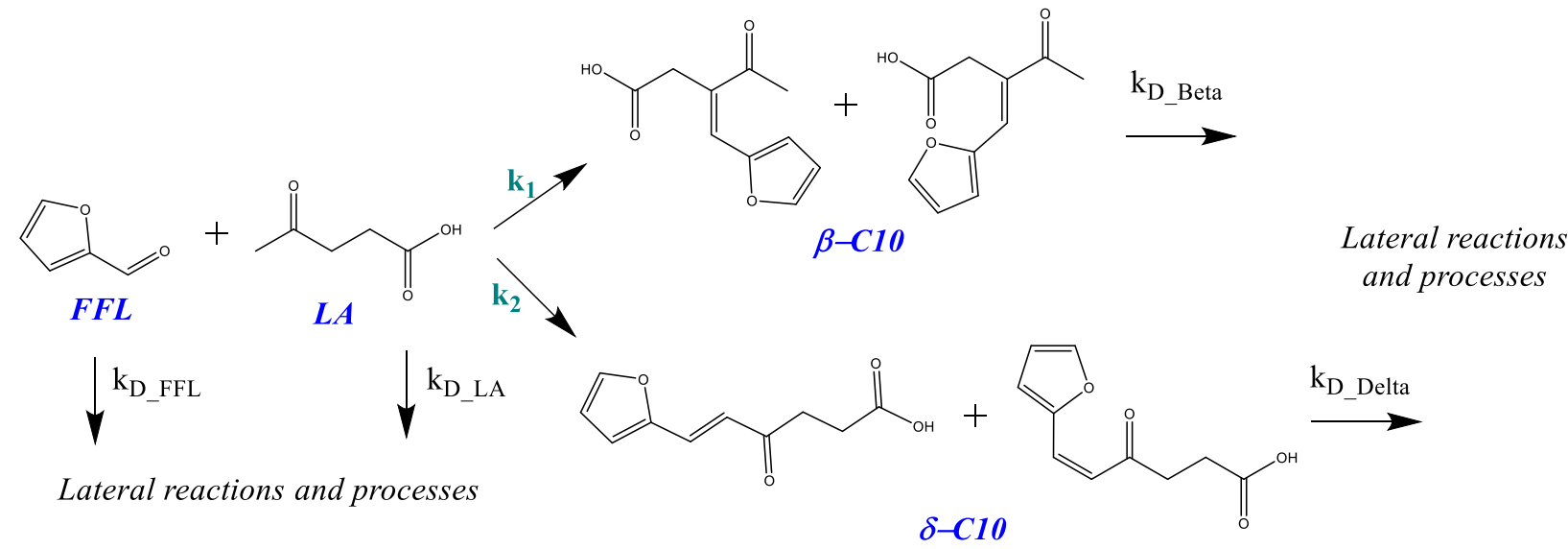

Scheme 1. Proposed reaction network for FFL-LA aldol condensation.

According to these results, the general acid aldolization mechanism is divided into two reaction pathways, as function of the site of proton abstraction, either on the tertiary carbon (producing the branched compound, $\beta-C 10)$, or on the terminal carbon. In this latter case, the linear product is obtained. These divergent routes are detailed in Scheme $\mathbf{2}$. The first step of this mechanism is the protonation of the carbonyl group of NaLe, by the interaction with the proton of the hydroxyl group present on the catalyst surface. ${ }^{46}$ The protonated species then undergo tautomerization, producing the subsequent enol. Two different compounds can be obtained, since the nucleophilic attack can concern two different $\alpha$ proton of the levulinate. Successively, 
these enols attack the carbonyl group of FFL, producing a $\beta$-hydroxy ketone ( $\mathrm{C} 10-\mathrm{OH})$. This intermediate is not observed because it undergoes fast dehydration, yielding the $\alpha-\beta$ unsaturated ketones ( $\beta$ - and $\delta$-C10). Since terminal enols are less stable, selectivity to the $\beta$ isomer is largely higher than the corresponding to $\delta$-isomer.

(a)
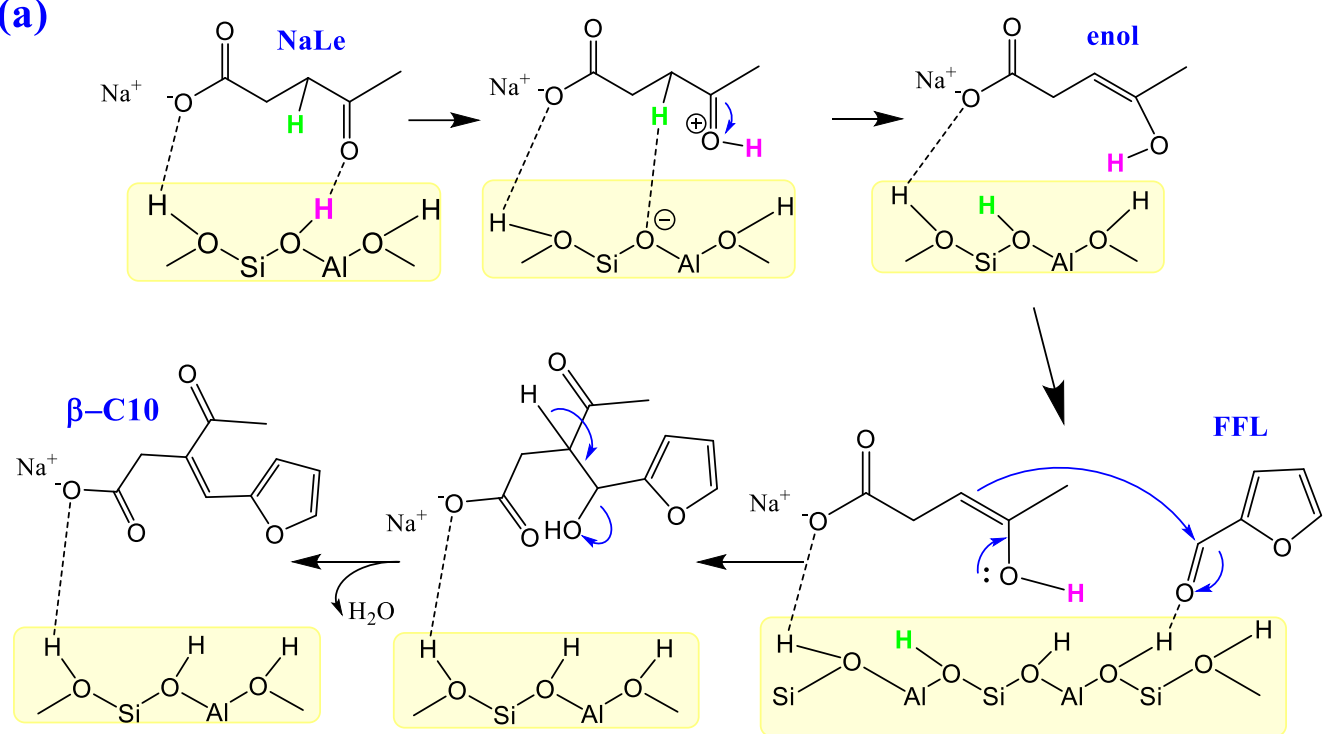

(b)
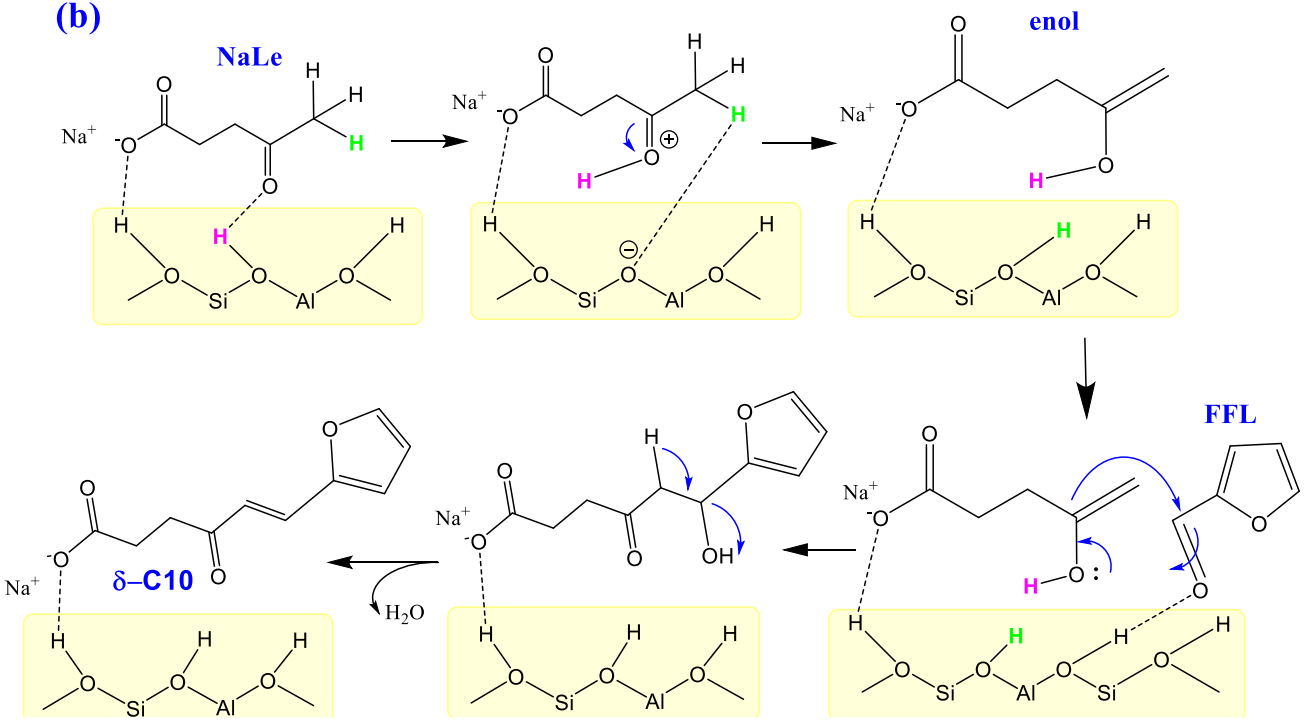

Scheme 2. Reaction mechanism of NaLe-FFL aldol condensation catalysed by acid zeolites to obtain (a) $\beta$-C10 and (b) $\delta$-C10. 
None of these steps directly involves the carboxylic group. Therefore, it is not evident why the reaction takes place when working with the NaLe but not with LA or EL. In aqueous medium, NaLe is totally dissociated into $\mathrm{Na}^{+}$and levulinate. As the reaction $\mathrm{pH}(6)$ is higher than the pKa of the LA (4.64), anion prevails over the protonated form, when the NaLe is used as reactant. In the case of LA, the situation is the opposite: as the $\mathrm{pH}$ of the reaction $(\mathrm{pH}=2.57)$ is lower than the pKa (4.64), so most of the levulinate is presented in its protonated form. We assume that the interaction between levulinate or levulinic acid and the catalytic surface is the key parameter in this discussion. Based on these facts, we propose that the adsorption of levulinic acid on the catalyst surface is too weak to initiate the reaction, while the adsorption of levulinate is co-operatively enhanced by the carboxylate anion. In good agreement with this hypothesis, the reaction with EL does not take place, since most of the levulinate obtained by the reverse reaction is directly protonated $(\mathrm{pH}=3.5)$. This hypothesis was tested by comparing the amount of LA and NaLe adsorbed on the ZSM-5. Working at the same initial concentration $\left(0.236 \mathrm{~mol} \cdot \mathrm{L}^{-1}\right)$, the amount of levulinate adsorbed on the zeolite is more than 7 times higher than the amount of LA adsorbed ( 0.9 and $5.7 \%$, respectively).

Considering the different distribution of both compounds, a predominant production of $\beta-\mathrm{C} 10$ is suggested, in good agreement with the higher stability of anions involving highly substituted carbons. This fact is emphasized by comparing the evolution of $\beta-\mathrm{C} 10 / \delta-\mathrm{C} 10$ ratio with the time. This magnitude decreases from infinity at the initial moments (no $\delta$-C10 is quantitatively detected during the first $20 \mathrm{~h}$ of reaction) to 21.5 after $24 \mathrm{~h}$. This evolution could be explained by two different or synergetic reasons. On the one hand, if both compounds are obtained on the same acid sites, longer times are required to promote the linear adduct (slower kinetics). 
On the other hand, if both products require different acidity strengths, then the first set of active sites are partially blocked during the reaction, enhancing the proportion of free acid sites involved in the formation of the linear compound.

In order to check the first hypothesis, a kinetic analysis had to be carried out, requiring studying the reaction at different temperatures. The influence of this parameter was study by analyzing the experimental results obtained at 393,423 and $448 \mathrm{~K}$ in comparison to the previous results at $373 \mathrm{~K}$, with Figure $\mathbf{2}$ comparing the results.

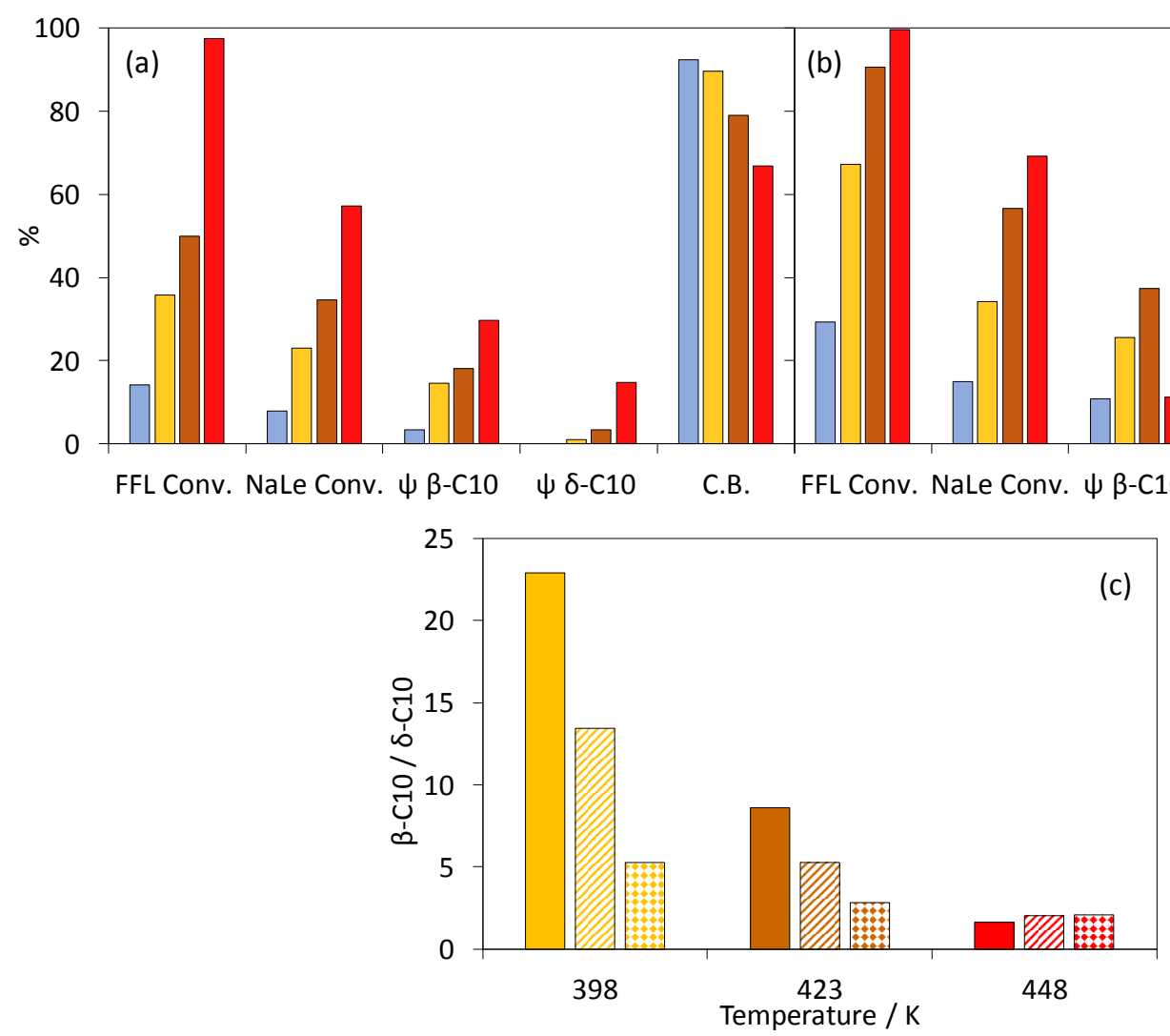

Figure 2. Summary of results obtained of NaLe-FFL aldol condensation catalysed by $1.8 \mathrm{~g}$ of ZSM-5 (23) as a function of the temperature and the reaction time (equimolar reactants ratio). Legend: (a) $8 \mathrm{~h}$, (b) $24 \mathrm{~h}$ and (c) products ratio at different times: $4 \mathrm{~h}$ (solid columns), $8 \mathrm{~h}$ (dashed columns) and $24 \mathrm{~h}$ (diamond columns); $373 \mathrm{~K}$ (blue); $398 \mathrm{~K}$ (yellow); $423 \mathrm{~K}$ (brown) and $448 \mathrm{~K}(\mathrm{red})$. 
As anticipated, a higher NaLe conversion is obtained as the temperature increases, reaching $69.2 \%$ after $24 \mathrm{~h}$ at $448 \mathrm{~K}$. As to the products, increasing total yields are obtained from 373 to $423 \mathrm{~K}$, regardless of the reaction time (from $10.9 \%$ to $37.3 \%$ in the case of $\beta-\mathrm{C} 10$, and from 0.5 $\%$ to $13.2 \%$ for $\delta$-C10). However, this trend is broken at the highest temperature tested (448 $\mathrm{K}$ ). At this temperature, a maximum of $45.5 \%$ total yield is obtained after $7 \mathrm{~h}$, whereas this magnitude decreases up to $16.6 \%$ when reaction time is extended to $24 \mathrm{~h}$. There is an increasing discrepancy between FFL conversion and the total yield obtained. Thus, almost total disappearance of FFL is observed at $448 \mathrm{~K}(99.6 \%$ conversion after $24 \mathrm{~h})$, whereas more than $80 \%$ of this conversion is not explained by the product yields. In the same way, $17.8,36.7$ and $40.1 \%$ of FFL conversions at 373,393 and $423 \mathrm{~K}$ are not transformed into the target compounds. These results discard the permanent adsorption without reaction (endothermic character of adsorption but positive role of temperature in oligomerization reactions), suggesting side reactions involving FFL leading to condensation. A high relevance of NaLe oligomerization is almost disregarded, since the difference between NaLe conversion and total yield is less than $6 \%$ except at the highest temperature.

In order to corroborate the presence of different side reactions as function of the temperature, the spent catalysts were analyzed by TPD, monitoring the evolution of individual signals of FFL, LA, $\delta-C 10$ and $\beta-C 10$ units (details in the Experimental Section). These results were compared with individual saturation of fresh catalysts with NaLe and FFL. All the TPD traces are plotted in the Supplementary Information (Figure S2), whereas the evolution of each adsorption is analyzed in Table 1 considering the values obtained at $373 \mathrm{~K}$ as baseline. Traces obtained at temperatures below $450 \mathrm{~K}$ mainly correspond to reactants signals, with a relative weight of 
products adsorption lower than $5 \%$. For FFL, the increase of the signal intensity observed as temperature increases corroborates the chemisorption, involving not only the adsorption but also oligomerization (promoted by the temperature). For the LA signal (direct correspondence with the NaLe one), a decrease in the adsorption is observed at low temperatures (congruent with physical adsorption), whereas there is an increase at the highest ones, suggesting oligomerizations. Results at $448 \mathrm{~K}$ clearly indicate a higher relevance of product signals; with the relative weight of $\delta$-C10 being more than 6 times higher than those obtained at milder reaction conditions. These results support the initial hypothesis of side reactions involving products at temperatures of $448 \mathrm{~K}$, Therefore, this temperature is discarded for future studies.

Table 1. TPD signal ratios as function of the reaction temperature taking the values of the lowest temperature as the baseline

\begin{tabular}{ccccc}
\hline T (K) & FFL (\%) & LA (\%) & $\boldsymbol{\beta}-$ C10 (\%) & $\boldsymbol{\delta}$-C10 (\%) \\
\hline 373 & 1.0 & 1.0 & 1.0 & 1.0 \\
398 & 2.7 & 0.6 & 1.2 & 1.9 \\
$\mathbf{4 2 3}$ & 3.0 & 1.7 & 3.4 & 4.7 \\
$\mathbf{4 4 8}$ & 3.5 & 2.6 & 3.8 & 6.0 \\
\hline
\end{tabular}

The influence of reaction temperature is also relevant in terms of reaction selectivity (Figure $2 c)$, which shows the ratio $\beta / \delta$ decreases from 21.5 at $373 \mathrm{~K}$ to 2.1 at $448 \mathrm{~K}$ as the temperature increases. In the TPD analyses, $\delta$-C10 signals are always higher than $\beta$-C10 ones (Fig. S2), i.e., in the opposite order than in liquid phase, suggesting that this isomer is preferentially adsorbed on the ZSM-5 surface. 
All these data were used to propose, for the first time in the literature, a kinetic model for NaLe-FFL aldol condensation. According to the temporal evolution of all the compounds involved, and in good agreement with the previous literature, a first order kinetic is proposed for the compound that suffers the enolization (NaLe). ${ }^{13,28,34,47}$ Previous studies of base catalyzed aldol condensations suggest that FFL is completely adsorbed on the surface, without any real influence on the kinetics (pseudo-zero order). ${ }^{13,28}$ This assumption cannot be made in this case, since interactions between FFL and zeolites can be different, which also introduces its reaction order as a variable. The kinetic model is laid out in Scheme 1. To sum up, two parallel reactions are considered, $k_{1}$ and $k_{2}$ being the kinetic constants for the formation of $\beta$ - and $\delta$-adducts, respectively. Four other constants ( $\mathrm{k}_{\mathrm{D}_{-} \mathrm{LA}}$ for $\mathrm{NaLe}, \mathrm{k}_{\mathrm{D}_{-}} \mathrm{FF}$ for $\mathrm{FFL}, \mathrm{k}_{\mathrm{D}_{-} \beta}$, and $\mathrm{k}_{\mathrm{D}_{-} \delta}$ for $\mathrm{C} 10 \mathrm{~s}$ ) represent the effects of the adsorption and oligomerization of reactants and products on the catalyst surface. The kinetic orders for these lateral processes are also included in the model. A differential equations system is proposed, assuming an ideal batch reactor.

$$
\begin{gathered}
\frac{d\left(\text { NaLe }_{1: 1}\right)}{d t}=-k_{1} \cdot C_{N a L e_{1: 1}} \cdot C_{F F L_{1: 1}}^{m}-k_{2} \cdot C_{N a L e_{1: 1}} \cdot C_{F F L_{1: 1}}^{m}-k_{D_{N a L e}} \cdot C_{N a L e_{1: 1}}^{n} \\
\frac{d\left(F F L_{1: 1}\right)}{d t}=-k_{1} \cdot C_{N a L e_{1: 1}} \cdot C_{F F L_{1: 1}}^{m}-k_{2} \cdot C_{N a L e_{1: 1}} \cdot C_{F F L_{1: 1}}^{m}-k_{D_{F F L}} \cdot C_{F F L_{1: 1}}^{p} \\
\frac{d\left(\beta-C 10_{1: 1}\right)}{d t}=k_{1} \cdot C_{N a L e_{1: 1}} \cdot C_{F F L_{1: 1}}^{m}-k_{D_{\beta}} \cdot C_{\beta-C 10_{1: 1}}^{q} \\
\frac{d\left(\delta-C 10_{1: 1}\right)}{d t}=k_{2} \cdot C_{N a L e_{1: 1}} \cdot C_{F F L_{1: 1}}^{m}-k_{D_{\delta}} \cdot C_{\delta-C 10_{1: 1}}^{r}
\end{gathered}
$$

As some of variables are related to kinetic orders, experiments with different initial reactant ratios had to be carried out. Assuming that most of the side reactions involve FFL, different excesses of NaLe were studied $(2: 1,5: 1)$, being the main results compared to those obtained 
with 1:1 in Figure 3. For these studies, temperature was fixed at $423 \mathrm{~K}$, this value being the optimum one obtained in the previous screening. The increase in final FFL conversion and the decrease in NaLe conversion were as expected, according to their conditions of limiting and excess reactant, respectively. The most relevant aspect is the evolution of the carbon balance, with a constant increase as NaLe excess increases (76.8, 91 and $97.1 \%$, at 1:1, 2:1, and 5:1, respectively). Thus, more than $40 \%$ of FFL converted at 1:1 is not transformed into products. This percentage is reduced to 25 and $0 \%$ when working at 2:1 and 5:1, respectively. However, at 5:1, $8 \%$ of NaLe converted is not observed in the reaction products, suggesting that a high excess of NaLe is also detrimental, as this promotes side reactions involving this acid. As to the products, the branched product is the main one under all the conditions tested, reaching the maximum selectivity at 2:1. At these conditions, the ratio $\beta / \delta$ increases from $2.8(1: 1)$ to 5.8 (2:1), decreasing to 3.4 when working at 5:1. In overall terms, working at 5:1 also implies a decrease in the total yield, reaching a maximum of $42.5 \%$, whereas $50 \%$ is obtained at $1: 1$ and 2:1.

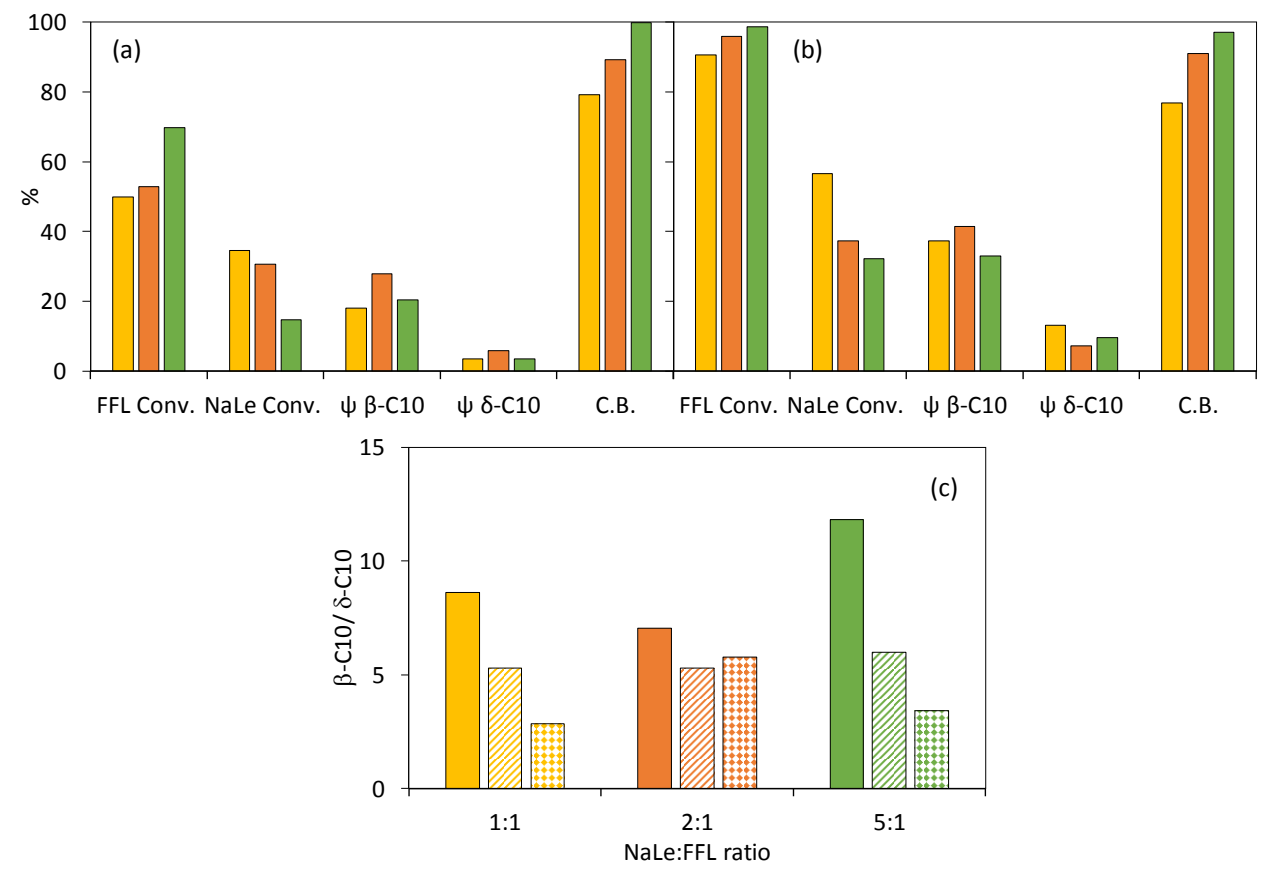

Figure 3. Summary of results obtained for the NaLe-FFL aldol condensation catalysed by $1.8 \mathrm{~g}$ of ZSM-5 (23) as a function of the reactants ratio and the reaction time $(423 \mathrm{~K})$. Legend: (a) $8 \mathrm{~h}$, (b) $24 \mathrm{~h}$ and (c) products ratio at different times: $4 \mathrm{~h}$ (solid columns), $8 \mathrm{~h}$ (dashed columns) and $24 \mathrm{~h}$ (diamond columns); $373 \mathrm{~K}$ (blue); 1:1 (yellow); 2:1 (orange) and 5:1 (green). 
These results were considered -together with the temperature influence-implemented in the kinetic model. The proposed model was solved numerically using MATLAB code, performing all the calculations and solving the set of ordinary differential equations ("ode45"). The fitting of the unknown parameters from the model is accomplished by the least-square method. The MATLAB function "Isqcurvefit" using the Levenberg-Marquardt algorithm was used. The coefficient of determination was calculated with MATLAB function "rsquare". Figure 4 shows the good fit of the proposed model, whereas Table $\mathbf{2}$ summarizes the values of the kinetic and adsorption constants for all the conditions studied, as well as the activation energy obtained by applying the Arrhenius equation. 

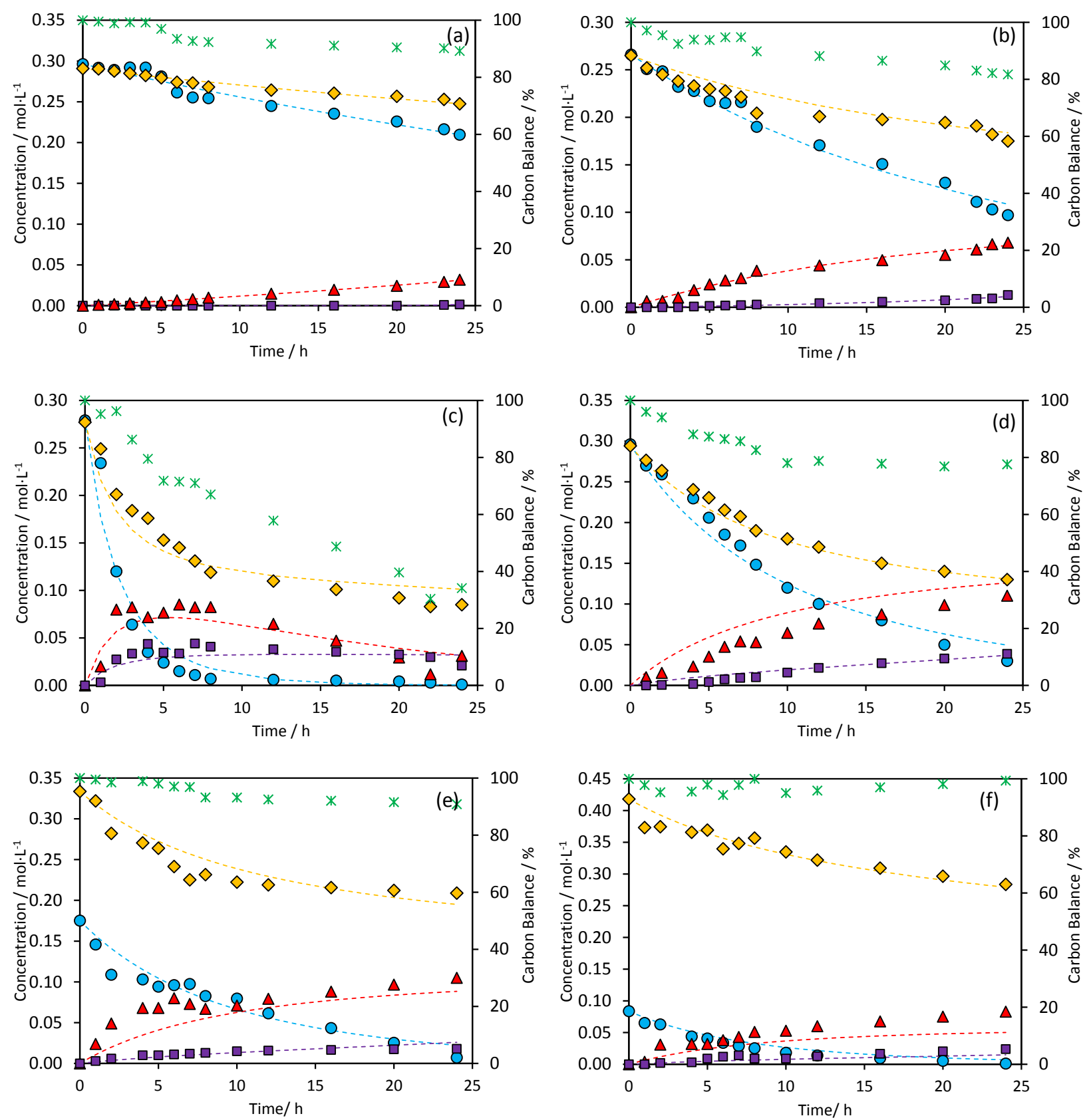

Figure 4. Kinetic fitting for different conditions studied in terms of temperature and NaLe:FFL ratio. Results correspond to: (a) $398 \mathrm{~K}, 1: 1$; (b) $403 \mathrm{~K}, 1: 1$; (c) $448 \mathrm{~K}, 1: 1$; (d) $423 \mathrm{~K}, 1: 1$; (e) $423 \mathrm{~K}$, 2:1; (f) $423 \mathrm{~K}, 5: 1$. Legend: (a) $398 \mathrm{~K}$; (b) $423 \mathrm{~K}$, and (c) $448 \mathrm{~K}$. Experimental points plotted with different symbols, whereas broken lines corresponds to kinetic model predictions. Symbols:

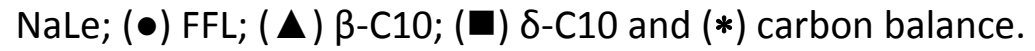


Table 2. Rate and adsorption constants for the fitting of the experimental results to the proposed kinetic model at different temperatures and $1.8 \mathrm{~g}$ of ZSM- 5 (23). $\mathrm{K}_{\mathrm{D}_{-} \beta}$ and $\mathrm{k}_{\mathrm{D}_{-} \delta}$ are only reported at the highest temperature, in other cases, this value is less than 0.001 . The activation energy for $\mathrm{k}_{\mathrm{D}_{-} \mathrm{LA}}, \mathrm{k}_{\mathrm{D}_{-} F F}, \mathrm{k}_{\mathrm{D}_{-} \beta}$ and $\mathrm{k}_{\mathrm{D}_{-} \delta}$ has not been calculated as these constants are not intrinsic kinetic parameters of the target reaction.

\begin{tabular}{|c|c|c|c|c|c|c|c|}
\hline & $\begin{array}{c}k_{1} \\
\left(L \cdot h^{-1} \cdot \mathrm{mol}^{-1}\right)\end{array}$ & $\begin{array}{c}k_{2} \\
\left(L \cdot h^{-1} \cdot \mathrm{mol}^{-1}\right)\end{array}$ & $\begin{array}{c}k_{D_{\text {_NaLe }}} \\
\left(\mathrm{L}^{1.5} \cdot \mathrm{h}^{-1} \cdot \mathrm{mol}^{-1.5}\right) \mathrm{k} 2\end{array}$ & $\begin{array}{l}k_{D_{D} F F L} \\
\left(h^{-1}\right) k 3\end{array}$ & $\begin{array}{l}k_{D \_\beta} \\
\left(h^{-1}\right)\end{array}$ & $\begin{array}{l}k_{D_{-} \delta} \\
\left(h^{-1}\right)\end{array}$ & $r^{2}$ \\
\hline $373 \mathrm{~K}$ & 0.013 & 0.000 & 0.026 & 0.013 & - & - & 0.999 \\
\hline $398 \mathrm{~K}$ & 0.076 & 0.003 & 0.038 & 0.026 & - & - & 0.992 \\
\hline $423 \mathrm{~K}$ & 0.198 & 0.036 & 0.056 & 0.043 & - & - & 0.985 \\
\hline $448 \mathrm{~K}$ & 0.725 & 0.249 & 0.273 & 0.271 & 0.054 & 0.003 & 0.958 \\
\hline $\begin{array}{c}\text { Ea }\left(\mathrm{kJ} \cdot \mathrm{mol}^{-1}\right) \\
\mathrm{r}^{2}\end{array}$ & $\begin{array}{c}72.7 \\
0.992\end{array}$ & $\begin{array}{l}128.0 \\
0.999\end{array}$ & - & - & - & - & - \\
\hline
\end{tabular}

In general terms, proposed model adequately fits the experiments performed at different reactant ratios and temperatures, obtaining a total determination coefficient of 0.982 . A first kinetic order of FFL was obtained for both direct reactions (experimental value of 0.94). This result indicates a weaker interaction between FFL and the zeolitic surface than when working with basic catalysts (zero-th order ${ }^{13,28}$ ). In good agreement with this hypothesis, side reactions involving this product disappear when increasing the NaLe:FFL ratio, whereas they were always relevant when working with basic conditions. ${ }^{13,28}$ In the same way, a first order kinetic for FFL was obtained for the lateral reactions involving FFL and both products, suggesting side processes concerning permanent adsorption with a limited oligomerization. Lateral reactions of both products are only relevant at the highest temperature tested (448 K). On the contrary, the side reaction of NaLe has a 2.5 order and a relevant kinetic constant. The high kinetic order obtained as well as the almost absence of real effects of this undesired reaction, except in the 
case of the highest levulinic excess (5:1), suggests that side reaction involving NaLe is a complex step not only determined by the concentration of NaLe but also by some intermediates not quantified in the liquid phase. This hypothesis requires more experiments to be corroborated (see Catalyst performance section).

The rate constants obtained follow a perfect Arrhenius dependence with the temperature, obtaining an activation energy for the branched product (C10) almost fifty percent lower than the one obtained for the linear adduct. This result explains the increasing differences observed in both profiles as the temperature decreases, with an almost total disappearance of C10 at temperatures lower than $393 \mathrm{~K}$.

\section{Catalyst performance of different zeolites}

Two main parameters are analysed in this section: the influence of acidity, by comparing the activity of two different ZSM-5 zeolites with different $\mathrm{SiO}_{2} / \mathrm{Al}_{2} \mathrm{O}_{3}$ ratio; and the influence of catalyst's structure, by comparing the activity of the ZSM-5 zeolite with BETA and Mordenite. In both cases, the study is focused on the activity, but also selectivity to the main reaction products. All reactions are carried out at $398 \mathrm{~K}$ with a NaLe:FFL initial ratio of 1:1.

$\underline{\text { Influence of surface acidity }}$

The role of surface acidity was studied comparing the activity of two ZSM-5 zeolites with different $\mathrm{SiO}_{2} / \mathrm{Al}_{2} \mathrm{O}_{3}$ ratio: 23 and 80 . Experimental results are analysed in Figure 5. 


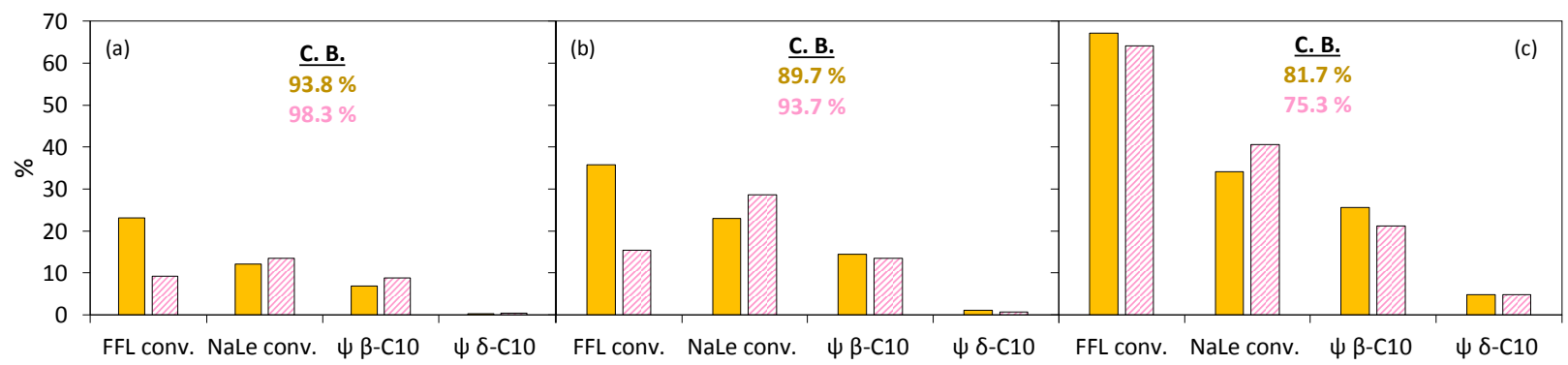

Figure 5. Summary of results obtained from the NaLe-FFL aldol condensation using equimolar reactants ratio at $398 \mathrm{~K}$ and catalysed by $1.8 \mathrm{~g}$ of ZSM-5 with different $\mathrm{SiO}_{2} / \mathrm{Al}_{2} \mathrm{O}_{3}$ ratio: 23 (solid columns) and 80 (dashed columns). Symbols: (a) $4 \mathrm{~h}$; (b) $8 \mathrm{~h}$ and (c) $24 \mathrm{~h}$.

The main differences are related to the disappearance of FFL at low reaction times $(4 \mathrm{~h})$ being more than three times higher in the case of the ZSM-5 (23). This fact is congruent with the existence of a higher concentration of acid sites, as it is reported in Table $\mathbf{3}$, and its promotion of adsorption processes. As anticipated, the different acidity is the main morphological difference between both materials, since both zeolites have the same framework structure (intersecting straight and sinusoidal channels by 10-membered oxygen ring). However, the different Si/Al distribution on the surface also affect to the external surface, being $25 \%$ higher in the case of the less acidic material. 
Table 3. Textural and acidic properties of zeolite materials. BET, surface area from BET method; $V_{\text {micro, }}$ microporevolume from t-plot method; $V_{\text {meso, }}$ mesopore volume (from BJH method);

$D_{\text {Howartz, }}$ average pore diameter for microporous materials.

\begin{tabular}{lcccccccc}
\hline Catalyst & $\begin{array}{c}\text { BET } \\
\left(\mathbf{m}^{2} \cdot \mathbf{g}^{-1}\right)\end{array}$ & $\begin{array}{c}\mathbf{S}_{\text {ext }} \\
\left(\mathbf{m}^{2} \cdot \mathbf{g}^{-1}\right)\end{array}$ & $\begin{array}{c}\mathbf{V}_{\text {micro }} \\
\left(\mathbf{c m}^{3} \cdot \mathbf{g}^{-1}\right)\end{array}$ & $\begin{array}{c}\mathbf{V}_{\text {meso }} \\
\left(\mathbf{c m}^{3} \cdot \mathbf{g}^{-1}\right)\end{array}$ & $\begin{array}{c}\text { DHowartz } \\
(\AA \mathbf{A})\end{array}$ & $\begin{array}{c}\text { Total acidity } \\
\left(\boldsymbol{\mu m o l ~} \mathbf{~ H H}_{3} \cdot \mathbf{g}^{-1}\right)\end{array}$ & $\begin{array}{c}\text { \% of weak } \\
\text { acidity } \\
\left(\mathbf{T}_{\text {des }<560 ~ K)}\right.\end{array}$ & $\begin{array}{c}\text { Total acidity } \\
\left(\mathbf{m e q H}^{+} \cdot \mathbf{g}^{-1}\right)\end{array}$ \\
\hline ZSM-5 (23) & 353 & 109 & 0.12 & 0.07 & 8.0 & 350.3 & 65.5 & 2.49 \\
ZSM-5 (80) & 440 & 199 & 0.12 & 0.06 & 8.2 & 17.31 & 85.4 & 0.26 \\
MOR (20) & 440 & 59 & 0.18 & 0.08 & 8.8 & 139.5 & 65.5 & 1.35 \\
BETA (25) & 620 & 243 & 0.18 & 0.73 & 10.2 & 210.0 & 72.6 & 1.69 \\
\hline
\end{tabular}

The reaction is more selective for the condensation products at short times with the ZSM-5 (80) with better correspondence between FFL and products evolutions. $\beta-\mathrm{C} 10$ is the main compound obtained with both materials (7.2 and $9.2 \%)$, whereas the yield of $\delta$-C10 is almost negligible after only $4 \mathrm{~h}(0.3$ and $0.4 \%$, for ZSM-5 (23) and ZSM-5 (80), respectively). All these experimental results are in good agreement with the rate constants obtained by the same method as before and are listed in Table 4. Opposite behaviors are observed at increasing times. Thus, the total products yield after $8 \mathrm{~h}$ is slightly higher for the most acidic zeolite (15.6\% vs. $14.2 \%)$, reaching a $17 \%$ higher yield after 24 h (30.5 \% vs. $26 \%)$. The selectivity between both adducts is also different, with the final mixture obtained with the ZSM-5 (80) being enriched in the linear one $(\beta / \delta$ final ratios of 5.2 and 4.4 , respectively). Similar evolutions of FFL conversion are obtained in both cases, with final conversions of 67 and $64 \%$. On the contrary, significant differences are observed with the levulinate: whereas there is almost a complete correspondence between its conversion and the product yields with ZSM-5 (23), there is more than $15 \%$ of discrepancy in the case of the ZSM-5 (80). All these experimental results are in good agreement with kinetic values obtained. Thus, the production of linear 
adduct is enhanced when decreasing the acidity ( $k_{2}$ is 2.7 times higher), whereas the branched one, identified with the $k_{1}$, slightly decreases (10\%). Moreover, FFL side reactions are promoted by the acidity, but this increase is negligible in comparison with the promotion of side reactions involving NaLe in the case of the ZSM-5 (80), as it is observed with a $\mathrm{k}_{\mathrm{D}_{-} \text {NaLe }} 7.4$ times higher than with the ZSM-5 (23).

Carbon balance closures obtained follow the expected evolution according to data previously discussed. At short reaction times (4 h), almost $100 \%$ is obtained with the ZSM-5 (80), whereas with the ZSM-5 (23) this parameter is close to $90 \%$ (93.8\%). On the contrary, opposite behaviors are observed at long reaction times. A continuous and gradual decrease to $82 \%$ is observed with the most acidic material. However, the carbon balance decreases to $75 \%$ with the ZSM-5 (80), this decrease being promoted only at long reaction times (it keeps almost constant over $94 \%$ during the first $8 \mathrm{~h}$ ). This behavior is congruent with the previous hypothesis that deactivation due to NaLe is not directly related to this compound but to a non-identified product involving this molecule (or other derivative obtained by a non-catalytic process) and the linear product $(\delta-C 10)$. This assumption is based on two pieces of evidence: on the one hand, the small difference between $\delta$-C10 with both materials does not correspond with the relevance difference in the rate constants, which is congruent with the existence of other steps involving $\delta-C 10$ as reactant. On the other hand, TPO analyses of spent catalyst suggests the presence of a totally different type of solids deposed on the catalytic surface of the ZSM-5 (80), as it is observed in Fig. S3. Traces obtained during the oxidation of ZSM-5 (23) have two main peaks, at 587 and $625 \mathrm{~K}$. The first one is also observed with the ZSM-5 (80), being assigned to FFL oligomers (common side reaction observed in both cases with higher relevance with the 
zeolite). However, the second peak does not appear in the TPO of ZSM-5 (80), being substituted by a main peak at $710 \mathrm{~K}$. This peak demonstrates the existence of a different reaction, not observed with the ZSM-5 (23).

This undesired reaction observed with the less acidic material suggests that the key parameter is not the total amount, but also the acid strength distribution of these sites. $\mathrm{NH}_{3}$-TPD analyses of fresh materials (Fig. S4) indicate the existence of two types of acid sites: weak and medium strength ones (desorption temperatures from 298 to $560 \mathrm{~K}$ for the weak acids, and from 560 to $820 \mathrm{~K}$ for medium sites). ZSM-5 (23) has a total concentration of acid sites 20 times higher than the one of ZSM-5 (80). In addition, $34.5 \%$ of these sites are medium strength sites, whereas this percentage decreases to less than $14 \%$ in the case of ZSM- 5 (80). It is well known that internal carbons are nucleophilic centers stronger than the terminal ones. According to this, the protons of these internal carbons are easily abstracted since they are more acidic than the terminal ones (always comparing only $\mathrm{C}-\mathrm{H}$ bonds). These protons play a key role once the carbonyl is protonated, since one of them must be abstracted from the levulinate to generate the enol and restore the electro-neutrality of the zeolitic material (tautomerization step). The proton abstraction must be catalyzed by a basic acid site (the free oxygen of the silanol group produced once the $\mathrm{H}$ has passed to the NaLe structure). The more electropositive hydrogen, the weaker basicity required to remove it, and the stronger conjugated acidity associated to it. According to this, the catalyst that enhances the production of the linear compound must have a higher percentage of weak acidity, since the conjugated stronger basic site is required to stabilize the intermediate obtained. On the contrary, removing the $\alpha$-hydrogen of the C3 position only requires medium-weak basicity, corresponding to medium acidity of the silanol 
group. In good agreement with this analysis, the $\beta / \delta$ ratio is higher with the zeolite enriched in medium-strength acidic sites, ZSM-5 (23), mainly at short times (286 vs. 51.7).

$\underline{\text { Structure influence }}$

Figure 6 shows the results obtained for the FFL-NaLe aldol condensation reaction after (a) $8 \mathrm{~h}$, and (b) $24 \mathrm{~h}$ of performing the aldol condensation at $398 \mathrm{~K}$ with three different zeolites: ZSM-5 (23); mordenite, labelled as MOR (20); and beta zeolite, identified as BETA (25).

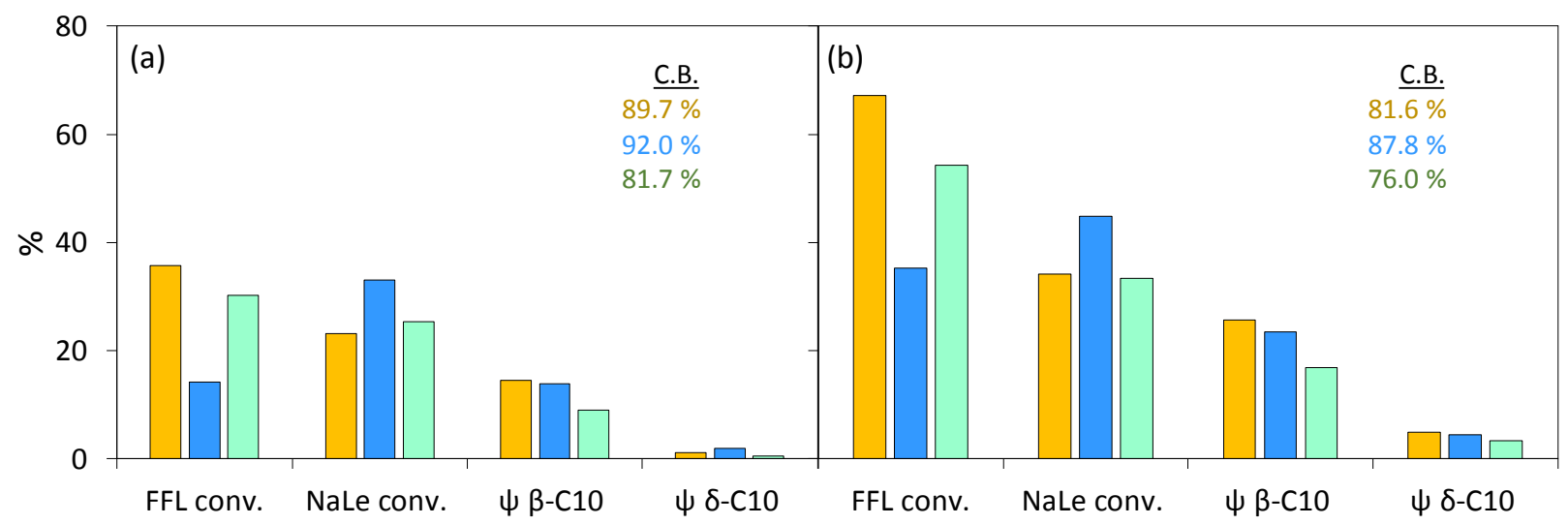

Figure 6. Summary of results obtained of NaLe-FFL aldol condensation at $398 \mathrm{~K}$ and catalysed by $1.8 \mathrm{~g}$ of different zeolites as function of the reaction time (equimolar reactants ratio): (a) $8 \mathrm{~h}$ and (b) 24h. Legend: ZSM-5 (23) (yellow); MOR (blue) and BETA (green).

In this case, conclusions drawn after $8 \mathrm{~h}$ can be directly applied to the analysis at $24 \mathrm{~h}$ (opposite situation than what happened with the acidity comparison). The lowest FFL conversion (35.2\%) is obtained with MOR, while the values for BETA and ZSM-5 are 54.2, $63.5 \%$, respectively. As to NaLe, the highest conversion is reached with MOR (44.8\%), whereas similar values where quantified for BETA and ZSM-5 (34.1 and 33.4\%, respectively). The maximum C10 total yield is obtained with ZSM-5 (30.5\%), very similar yield than the corresponding value with MOR (27.9\%). On the contrary, the total yield obtained with BETA is significantly lower (20.3\%) than 
the one obtained with ZSM-5. This value is $33 \%$ lower than the obtained with ZSM-5, despite giving similar conversions of both reactants. In all the cases, the branched adduct $(\beta-C 10)$ is obtained in higher amounts than the linear one $(\delta-C 10)$, these differences being more pronounced at short reaction times. Thus, initial $\beta / \delta$ ratios of 286,249 and 21 for ZSM-5, MOR and BETA during the first two hours decrease to 5.2, 5.1 and 5.4 after $24 \mathrm{~h}$, respectively. These results are in good agreement with the percentage of weak acid sites (strong conjugated bases) of these materials, as observed in Table 3. According to these results, the best selectivity for desired compounds seems to be observed with the MOR, with side reactions taking place to a lower extent. Considering the calculated kinetic constants (see Table 4), this material almost prevents FFL side reactions ( $\mathrm{K}_{\mathrm{D}_{-} \mathrm{FFL}} 13$ times lower than the ones obtained with ZSM-5 and BETA). This value is in good agreement with the low FFL disappearance not explained by the C10 formation (more than 5 times lower than values with the other materials).

Table 4. Rate and adsorption constants for the fitting of the experimental results to the proposed kinetic model using different zeolites at $398 \mathrm{~K}$ and the values for MOR at different temperatures. $\mathrm{K}_{\mathrm{D}_{\_} \beta}$ and $\mathrm{k}_{\mathrm{D}_{-} \delta}$ are only reported at the highest temperature, in other cases, this value is less than 0.001 . Activation energy for $\mathrm{k}_{D_{-} N a L e}, \mathrm{k}_{D_{-}} F F_{L}$ and $\mathrm{k}_{\mathrm{D}_{-} \beta}$ and $\mathrm{k}_{\mathrm{D}_{-} \delta}$ has not been calculated as these constants are not intrinsic kinetic parameters.

\begin{tabular}{|c|c|c|c|c|c|c|c|c|}
\hline Catalyst & $\mathrm{T}(\mathrm{K})$ & $\begin{array}{c}k_{1} \\
\left(L \cdot h^{-1} \cdot \mathrm{mol}^{-1}\right)\end{array}$ & $\begin{array}{c}k_{2} \\
\left(L \cdot h^{-1} \cdot \mathrm{mol}^{-1}\right)\end{array}$ & $\begin{array}{c}\mathrm{K}_{\mathrm{D}_{-} \text {NaLe }} \\
\left(\mathrm{L}^{1.5} \cdot \mathrm{h}^{-1} \cdot \mathrm{mol}^{-}\right. \\
\left.{ }^{1.5}\right) \mathrm{k} 2\end{array}$ & $\begin{array}{l}k_{D_{2} F L L} \\
\left(h^{-1}\right) k 3\end{array}$ & $\begin{array}{l}k_{D_{\_} \beta} \\
\left(h^{-1}\right)\end{array}$ & $\begin{array}{l}k_{D_{-} \delta} \\
\left(h^{-1}\right)\end{array}$ & $r^{2}$ \\
\hline ZSM-5 (23) & & 0.076 & 0.003 & 0.038 & 0.026 & - & - & 0.992 \\
\hline ZSM-5 (80) & 398 & 0.067 & 0.008 & 0.125 & 0.022 & - & - & 0.992 \\
\hline \multirow[t]{2}{*}{ BEA (25) } & & 0.053 & 0.006 & 0.107 & 0.027 & - & - & 0.995 \\
\hline & 398 & 0.064 & 0.009 & 0.184 & 0.003 & - & - & 0.991 \\
\hline \multirow[t]{2}{*}{ MOR (20) } & 423 & 0.285 & 0.068 & 0.202 & 0.011 & - & - & 0.970 \\
\hline & 448 & 0.637 & 0.101 & 0.257 & 0.019 & 0.049 & 0.013 & 0.941 \\
\hline \multicolumn{2}{|c|}{$\begin{array}{c}\mathrm{Ea}\left(\mathrm{KJ} \cdot \mathrm{mol}^{-1}\right) \\
\mathrm{r}^{2}\end{array}$} & $\begin{array}{c}68.3 \\
0.981\end{array}$ & $\begin{array}{c}72.1 \\
0.892\end{array}$ & - & - & & - & - \\
\hline
\end{tabular}


These kinetic constant values are obtained applying the same mechanism proposed for the ZSM-5, supporting the hypothesis of same reaction pathway despite the catalytic structure used. The goodness of this fit is shown in Figure 7.
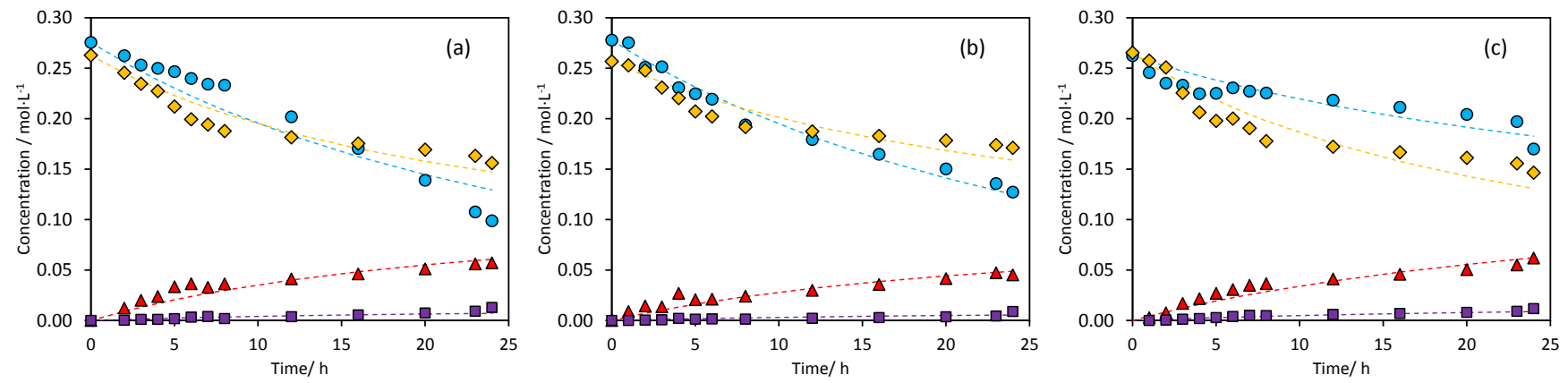

Figure 7. Kinetic model predictions for NaLe:FFL (1:1) condensation at $423 \mathrm{~K}$ as function of the catalyst. Results correspond to: (a) ZSM-5 (80); (b) BETA; (c) MOR. Experimental points plotted with different symbols, whereas broken lines corresponds to kinetic model predictions.

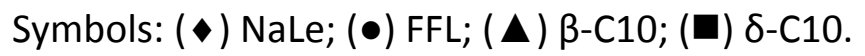

BETA zeolite was discarded because of the low final yields, but the goodness of the fit for MOR is not so clear, due to the high $\mathrm{k}_{\mathrm{D}_{-} \text {NaLe }}$ obtained (almost 11 times higher than with the ZSM-5). In good agreement with this value, the amount of NaLe not converted into C10s is almost 5 times higher than the one with ZSM-5.

In order to check if the negative consequences of these side reactions, previously suggested in the acidity study, prevail over the positive effect of almost preventing FFL oligomerization, reactions at different temperatures with MOR have been carried out. The main results after $24 \mathrm{~h}$ are compared in Figure 8, whereas the temporal evolution, as well as the kinetic prediction is included in the Supplementary Information (Figure S5). Results at $448 \mathrm{~K}$ are discarded according to the low carbon balance closure (33.6\%). However, values obtained at $423 \mathrm{~K}$ are 
significantly worse than those obtained with ZSM-5 (23), obtaining a carbon balance of $61 \%$ (compared to $77.5 \%$ with the ZSM-5). This carbon balance is explained by the lateral reactions involved NaLe (high increase in the disappearance of this reactant despite the decrease in the C10 yields) and the higher selectivity to $\delta-C 10$ adduct ( $\beta / \delta$ ratio of 14 , whereas a value of 25 was obtained with the ZSM-5). In summary, ZSM-5 is considered to be the best material since its higher activation energy for the linear adduct production (128 vs. $\left.72.1 \mathrm{~kJ} \cdot \mathrm{mol}^{-1}\right)$ prevents a

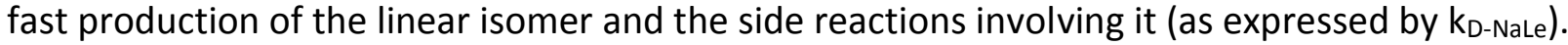

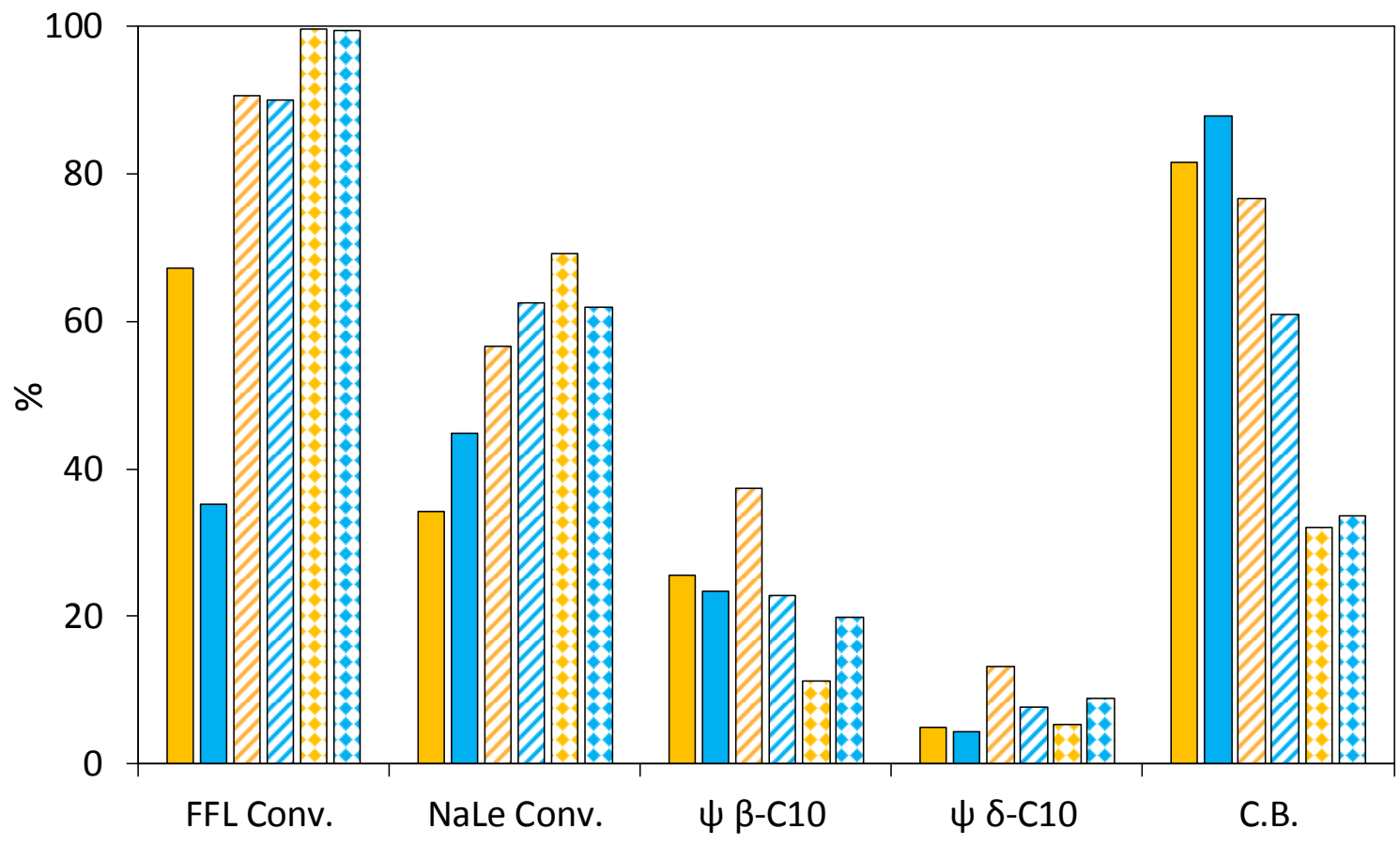

Figure 8. Comparison of results obtained of NaLe-FFL aldol condensation at $398 \mathrm{~K}$ and catalysed by $1.8 \mathrm{~g}$ of different zeolites as function of the reaction time (equimolar reactants ratio, $24 \mathrm{~h}$ ). Legend: ZSM-5 (23) (yellow) and MOR (20) (blue); $398 \mathrm{~K}$ (solid bars), $423 \mathrm{~K}$ (dashed bars) and $448 \mathrm{~K}$ (diamond bars)

The different behaviours of these three zeolites cannot be explained as function of their morphological properties since there is not any clear correlation between external surface, 
total one, or pore volume with the activity results (Table 3). Therefore, it can be concluded that this reaction is not sensitive to the zeolite structure. Instead, the distribution of the acid sites is the key parameter. Thus, an increase in the percentage of weak acid sites has a negative effect on the overall outcomes. There is a linear correlation between $k_{1}$ and $k_{2}$ and this type of acid sites with opposite trends (they promote the production of linear adduct, preventing the branched one). In good agreement, a clear decrease in the $\beta / \delta$ ratio with this acidity is obtained. On the contrary, the lateral reactions involving NaLe increase with the percentage of weak acid sites, whereas there is not any clear trend concerning the lateral reactions involving FFL. Figure 9 illustrate these evolutions.

As a summary of the previous analysis, the best conditions require working with ZSM-5 (23) at $423 \mathrm{~K}$. While side reactions involving NaLe are minimized, these conditions also favoured FFL oligomerization because of the high amount of medium-strength acid sites. Consequently, a low excess of LA is required to obtain the optimum activity $(2: 1)$ as it was previously demonstrated.

\section{Catalyst stability}

Stability tests were carried out employing the optimum conditions previously determined ( $24 \mathrm{~h}$, 423 K, 2:1 NaLe:FFL reactant ratio and ZSM-5 (23) catalyst). After each cycle the catalyst was recovered by filtration, washed with water and dried overnight at $373 \mathrm{~K}$, without applying any further regeneration procedure between cycles. Figure 10 shows the main results after four cycles. 


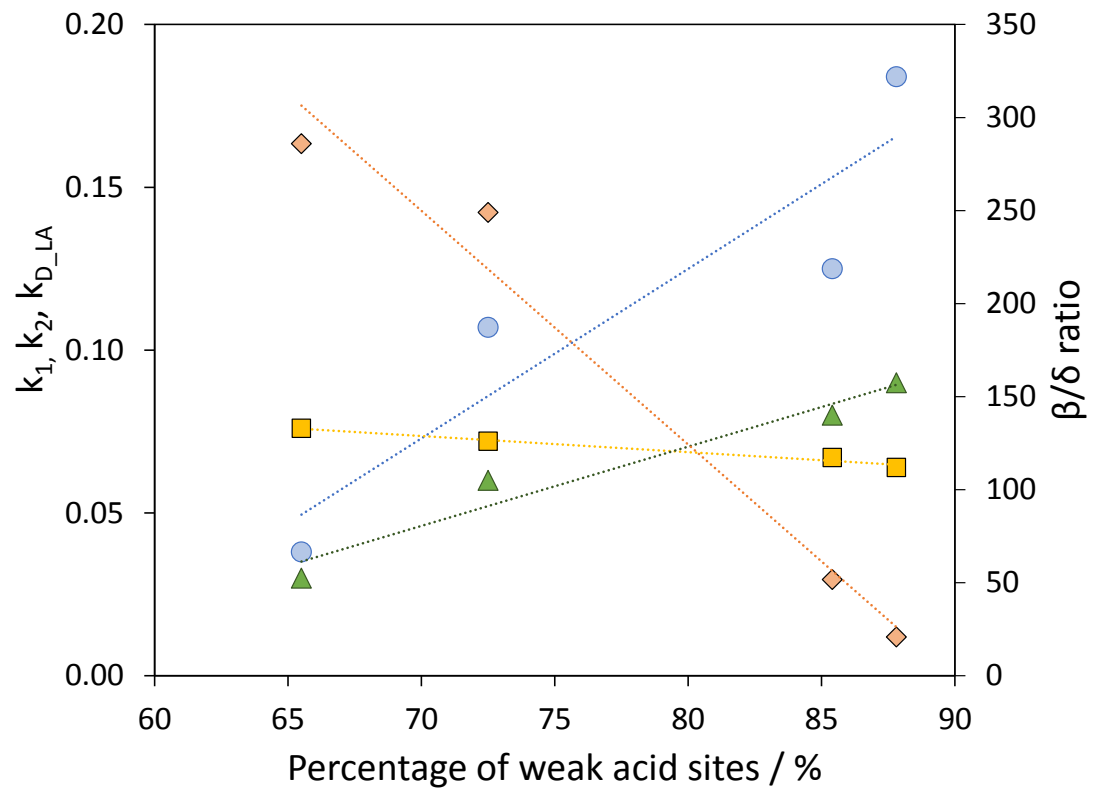

Figure 9. Evolution of different parameters with the relative percentage of weak acid sites presents in each material. Symbols: $(\boldsymbol{\square}) \mathrm{k}_{1} ;(\boldsymbol{\Delta}) \mathrm{k}_{2}(x 10) ;(\bullet) \mathrm{k}_{\mathrm{D}_{-} \mathrm{NaLe}}(\bullet) \beta / \delta$ ratio at initial times. A decrease of less than $10 \%$ in FFL conversion is observed (95 vs. $89 \%$, after the first and fourth cycle, respectively). Almost $60 \%$ of the total C10 obtained in the first cycle are still produced in the fourth one, with the $\beta$-C10 adduct suffering a larger decrease in its productivity. A gradual, but continuous decrease in the carbon balance closure $(87.5 \%$ after the fourth cycle) as well as the increasing discrepancy between reactant conversions and products yields is congruent with the deposition of these adducts as the main deactivation cause. At this point, it should be noted that the deactivation is markedly lower than in other reported condensation reaction, and that the deactivated catalyst can be fully regenerated by air treatment. TPO results also show that carbonaceous can be burned off below $800 \mathrm{~K}$, with the structure of the ZSM-5 being stable at this temperature. 


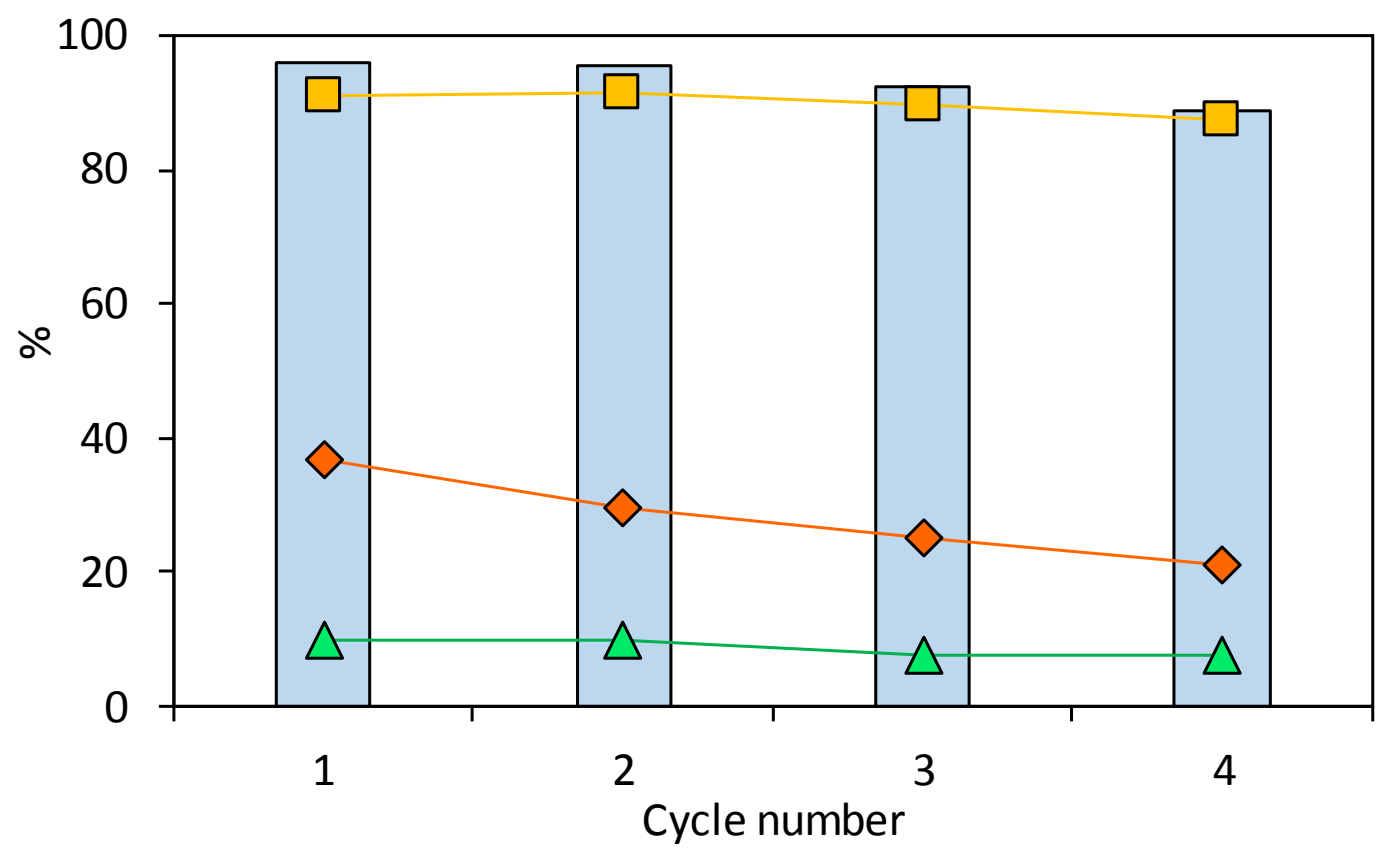

Figure 10. Reusability results after $24 \mathrm{~h}$ of each cycle at $423 \mathrm{~K}$, using ZSM-5 (23) and 2:1 as NaLe:FFL ratio. Legend: (blue bars) FFL conversion; ( $\mathbf{\square})$ Carbon Balance; ( $\bullet$ ) $\beta$-C10 yield; ( $\mathbf{\Delta}$ ) $\delta$ - C10 yield.

\section{CONCLUSIONS}

Promising results are obtained in the furfural and levulinic acid aldol condensation catalysed with acid zeolites. A prior neutralization of the carboxylic group of LA is required to prevent the cyclization, obtaining good activity results when using $\mathrm{Na}_{2} \mathrm{CO}_{3}$, obtaining the sodium levulinate. A deep study of reaction conditions was carried out, concluding that optimum values correspond to $423 \mathrm{~K}$, and an initial NaLe:FFL ratio 2:1. ZSM-5 (23) is identified as the best catalyst tested, realizing more than $50 \%$ of total yield (two C10 products obtained) with a good carbon balance closure (92\%). A kinetic model is proposed, suggesting furfural oligomerization 
as the main deactivation cause, as well as a negative interaction between NaLe excess and a high production of the linear adduct $(\delta-C 10)$. The acidity strength is identified as the key parameter to prevent this side reaction, being directly related to the prevalence of weak acid sites.

\section{ASSOCIATED CONTENT}

\section{Supporting Information}

The Supporting Information is available at

It includes: Fig. S1: m/z spectra of each compound involved in the reaction; Fig. S2: TPD analyses of spent catalyst; Fig. S3: TPO analyses of spent catalysts; Fig. S4: $\mathrm{NH}_{3}$-TPD of different zeolites; and Fig. S5: the temporal evolution and kinetic prediction of results obtained with MOR at different temperatures.

\section{AUTHOR INFORMATION}

\section{Corresponding Author}

*e-mail: sordonez@uniovi.es, Tel: +34 985103 437; Fax: + 34985103434 


\section{Author Contributions}

The manuscript was written through contributions of all authors. All authors have given approval to the final version of the manuscript.

\section{Funding Sources}

This work has been financed by the Spanish Ministry of Economy and Competitiveness (CTQ201789443-C3-2-R) and the Regional Government of Asturias (project reference IDI/2018/000116).

\section{REFERENCES}

(1) Hilali, S.; Fabiano-Tixier, A.S.; Ruiz, Z.; Hejiaj, Al.; Nouh, F.A.; Idlimam, A.; Bily, A.; Mandi, L.; Chemat, F., Green extraction of essential oils, polyphenols and pectins from orange peel employing solar energy: toward a zero-waste biorefinery. ACS Sust. Chem. Eng. 2019, 7, 11815-11822. Doi: 10.1021/acssuschemeng.9b02281.

(2) Han, X.; Guo, Y.; Liu, X.; Xia, Q.; Wang, Y., Catalytic conversion of lignocellulosic biomass into hydrocarbons: A mini review. Catal. Today 2019, 319, 2-13. Doi:

10.1016/j.cattod.2018.05.013.

(3) Malkar, R.S.; Daly, H.; Hardacre, C.; Yadav, G.D., Aldol condensation of 5hydroxymethylfurfural to fuel precursors over novel aluminum exchanged-DTP@ZIF-8. ACS Sust. Chem. Eng. 2019, 7, 16215-16224. Doi: 10.1021/acssuschemeng.9b02939. 
(4) Yutthalekha, T.; Suttipat, D.; Salakhum, S.; Thivasasith, A.; Nokbin, S.; Limtrakul, J.; Wattankit, C., Aldol condensation of biomass-derived platform molecules over aminegrafted hierarchical FAU-type zeolite nanosheets (Zeolean) featuring basic sites. Chem. Commun. 2017, 53, 12185-12188. Doi: 10.1039/c7cc06375j.

(5) Barrett, C.J.; Chheda, J.N.; Huber, G.W.; Dumesic, J.A., Single-reactor process for sequential aldol-condensation and hydrogenation of biomass-derived compounds in water. Appl. Catal. B 2006, 66, 111-118. Doi: 10.1016/j.apcatb.2006.03.001.

(6) Chheda, J.N.; Dumesic, J.A., An overview of dehydration, aldol-condensation and hydrogenation processes for production of liquid alkanes from biomass-derived carbohydrates. Catal. Today 2007, 123, 59-70. Doi: 10.1016/j.cattod.2006.12.006.

(7) Dowson, G:R.M.; Haddow, M.F.; Lee, J.; Wingad, R.L.; Wass, D.F., Catalytic conversion of ethanol into an advanced biofuel: unprecedented selectivity for n-butanol. Angew. Chem. Int. Ed. 2013, 52, 9005-9008. Doi: 10.1002/anie.201303723.

(8) Silks, L.A.; Gordon, J.C.; Wu, R.; Hanson, S.K., Process for preparation of furan derivatives by carbon chain extension through aldol reaction. WO2011022042A1, 2011.

(9) Yadav, G.D.; Yadav, A.R., Novelty of Claisen-Schmidt condensation of biomass derived furfural with acetophenone over solid super base catalyst. RSC Adv. 2014, 4, 6377263778. Doi: 10.1039/c4ra09194a. 
(10) Rapeyko, A.; Arias, K.S.; Climent, M.J.; Corma, A.; Iborra, S., Polymers from biomass: one pot two-step synthesis of furilydenepropanenitrile derivatives with MIL-100(Fe) catalyst. Catal. Sci. Technol. 2017, 7, 3008-3016. Doi: 10.1039/c7cy00463j.

(11) Siewert, R.; Zaitsau, D.H.; Emel'yanenko, V.N.; Verevkin, S.P., Biomass valorization: thermodynamics of the Guerbet condensation reaction. J. Chem. Eng. Data 2019 (in press). Doi: 10.1021/acs.jced.9b00419.

(12) Gordon, J.C.; Silks, L.A.; Sutton, A.D.; Wu, R., Schalf, M.; Waldie, F.; West, R.; Collias, D.I., Compounds and methods for the production of long chain hydrocarbons from biological sources. WO2013040311A1, 2013.

(13) Cueto, J.; Faba, L.; Díaz, E.; Ordóñez, S., Performance of basic mixed oxides for aqueousphase 5-hydroxymethylfurfural-acetone aldol condensation. Appl. Catal. B 2017, 201, 221-231. Doi: 10.1016/j.apcatb.2016.08.013.

(14) Xu, J.; Li, L.; Li, G.;Wang, A; Cong, Y.; Wang, X.; Li, N., Synthesis of renewable C8-C10 alkanes with angelica lactone and furfural from carbohydrates. ACS Sust. Chem. Eng. 2018, 6, 6126-6134. Doi: 10.1021/acssuschemeng.7b04797.

(15) Kikhtyanin, O.; Kubicka, D.; Cejka, J., Toward understanding of the role of Lewis acidity in aldol condensation of acetone and furfural using MOF and zeolite catalyst. Catal. Today 2015, 243, 158-162. Doi: 10.1016/j.cattod.2014.08.016. 
(16) Xiang, M.; Liu, J.; Fu, W.; Tang, T.; Wu, D., Improved activity for cellulose conversion to levulinic acid through hierarchization of ETS-10 zeolite. ACS Sust. Chem. Eng. 2017, 5, 5800-5809. Doi: 10.1021/ acssuschemeng.7b00529.

(17) Yan, K.; Jarvis, C.; Gu, J.; Yan, Y., Production and catalytic transformation of levulinic acid: A platform for specialty chemicals and fuels. Renew. Sust. Energ. Rev. 2015, 51, 986-997. Doi: 10.1016/j.rser.2015.07.021.

(18) Pileidis, F.D.; Titirici, M.M., Levulinic acid biorefineries: new challenges for efficient utilization of biomass. ChemSusChem 2016, 9, 562-582. Doi: 10.1002/cssc.201501405.

(19) Mellmer, M.A.; Gallo, J.M.R.; Martín Alonso, D.; Dumesic, J.A., Selective production of levulinic acid from furfuryl alcohol in THF solvent systems over H-ZSM-5. ACS Catal., 2015, 5, 3354-3359. Doi: 10.1021/acscatal.5b00274.

(20) Orlowski, I.; Douthwaite, M.; Iqbal, S.; Hayward, J.S.; Davies, T.E.; Bartley, J.K.; Miedziak, P.J.; Hirayama, J., The hydrogenation of levulinic acid to $\gamma$-valerolactone over Cu-ZrO2 catalysts prepared by a pH-gradient methodology. J. Energ. Chem. 2019, 36, 15-24. Doi: 10.1016/j.jechem.2019.01.015.

(21) Murugesan, K.; Aslhammari, A. S.; Sohail, M.; Jagadeesh, R. V., Levulinic acid derived reusable cobalt-nanoparticles-catalyzed sustainable synthesis of $\gamma$-valerolactone. ACS Sust. Chem. Eng. 2019, 7, 14756-14764. Doi: 10.1021/acssuschemeng.9b02692. 
(22) Lilga, M.A.; Padmaperuma, A.B.; Auberry, D.L.; Job, H.M.; Swita, M.S., Ketonization of levulinic acid and valerolactone to hydrocarbon fuel precursors. Catal. Today 2018, 302, 80-86. Doi: 10.1016/j.cattod.2017.06.021.

(23) Horváth, I.; Mehdi, H.; Fábos, V.; Mika, L.T., Valerolactone - a sustainable liquid for energy and carbon-based chemicals. Green Chem. 2008, 10, 238-242.

(24) Schuette, H.A.; Thomas, R.W., Normal valerolactone III. Its preparation by the catalytic reduction of levulinic acid with hydrogen in the presence of platinum oxide. JACS, 1930, $52,3010$.

(25) Faba, L.; Díaz, E.; Ordóñez, S., Base-catalyzed condensation of levulinic acid: a new biorefinery upgrading approach. ChemCatChem 2016, 8, 1490-1494. Doi: $10.1002 /$ cctc/201600064.

(26) Amarasekara, A.S.; Ha, U.; Fonari, M.S.; Bejagam, S.N.; Margetic, D., Sulfuric acid and amberlyst- $\mathrm{H}^{+}$catalyzed condensation reactions of renewable keto acids with paraformaldehyde: synthesis of a new dispiro bis-lactone ring system 2,9,13trioxadispiro[4.1.4.3]tetradecane-3,6,10-trione. RSC Adv. 2017, 7, 23917-23923. Doi: 10.1039/c7ra02303k.

(27) Li, Z.; Zhang, J.; Nielsen, M.M.; Wang, H.; Chen, C.; Xu, J.; Wang, Y.; Deng, T.; Hou, X., Efficient C-C bond formation between two levulinic acid molecules to produce C-10 compounds with the cooperation effect of Lewis and Bronsted acids. ACS Sust. Chem. Eng. 2018, 6, 5708.5711. Doi: 10.1021/acssuschemeng.7b04621. 
(28) Faba, L.; Díaz, E.; Ordóñez, S., Aqueous-phase furfural-acetone aldol condesnation over basic mixed oxides. Appl. Catal. B 2012, 113-114, 201-211. Doi:

10.1016/j.apcatb.2011.11.039.

(29) Wang, W.; Li, N.; Li, G.; Li, S.; Wang, W.; Wang, A.; Cong, Y.; Wang, X.; Zhang, T., Synthesis of renewable high-density fuel with cyclopentanone derived from hemicellulose. ACS Sust. Chem. Eng. 2017, 5, 1812-1817. Doi: 10.1021/ acssuschemeng.6b02554.

(30) Pagán-Torres, Y. J.; Wang, T.; Gallo, J. M. R.; Shanks, B. H.; Dumesic, J. A., Production of 5hydroxymethylfurfural from glucose using a combination of Lewis and Brønsted acid catalysts in water in a biphasic reactor with an alkylphenol solvent. ACS Catal. 2012, 2, 930-934. Doi: doi.org/10.1021/cs300192z.

(31) Bhanja, P.; Modak, A.; Chatterjee, S.; Bhaumik, A., Bifuncionalized mesoporous SBA-15: a new heterogeneous catalyst for the facile synthesis of 5-hydromethylfurfural. ACS Sust. Chem. Eng. 2017, 5, 2763-2773. Doi: 10.1021/ acssuschemeng.6b03100.

(32) Garcés, D.; Faba, L.; Díaz, E.; Ordóñez, S., Aqueous-phase transformation of glucose into hydroxymethylfurfural and levulinic acid by combining homogeneous and heterogeneous catalysis. ChemSusChem 2019, 12, 924-934. Doi: 10.1002/cssc.201802315.

(33) Huang, X.; Kudo, S.; Sperry, J.; Hayashi, J., Clean synthesis of 5- hydroxymethylfurfural and levulinic acid by aqueous Phase conversion of levoglucosenone over solid acid catalysts. ACS Sust. Chem. Eng. 2019, 7, 5892-5899. oi: 10.1021/acssuschemeng.8b05873. 
(34) Cueto, J.; Faba, L.; Díaz, E.; Ordóñez, S., Enhancement of furfural-cyclopentanone aldol condensation using binary water-ethanol mixtures as solvent. J. Chem. Technol.

Biotechnol. 2018, 93, 1563-1571. Doi: 10.1002/jctb.5522.

(35) Faba, L.; Díaz, E.; Ordóñez, S., Improvement on the catalytic performance of Mg-Zr mixed oxides for furfural-acetone aldol condensation by supporting on mesoporous carbons. ChemSusChem 2013, 6, 463-473. Doi: 10.1002/cssc.201200710.

(36) Desai, D. S.; Yadav, G. D., Green synthesis of furfural acetone by solvent-free aldol condensation of furfural with acetone over La203-MgO mixed oxide catalyst. ACS Sust. Chem. Eng. 2019, 58, 16096-16105. Doi: 10.1021/acssuschemeng.8b05873.

(37) Amarasekara, A.S.; Singh, T.B.; Larkin, E.; Hasan, M.A.; Fan, H.-J., NaOH catalyzed condensation reactions between levulinic acid and biomass derived furan-aldehydes in water. Ind. Crops. Prod. 2015, 65, 546-549. Doi: 10.1016/j.indcrop.2014.10.005.

(38) Liang, G.; Wang, A.; Zhao, X.; Lei, N.; Zhang, T., Selective aldol condensation of biomassderived levulinic acid and furfural in aqueous-phase over $\mathrm{MgO}$ and $\mathrm{ZnO}$. Green. Chem. $2016,18,3430-3438$.

(39) Scanlon, J.T.; Willis, D.E., Calculation of flame ionization detector relative response factors using the effective carbon number concept. J. Chromatogr. Sci. 1985, 23, 333-340. Doi: 10.1093/chromsci/23.8.333. 
(40) De Melo Lima, T.; Weber Paixao, M.; Correa, A.G., Angelica lactones: from biomassderived platform chemicals to value-added products. ChemSusChem 2018, 11, 24-47. Doi: 10.1002/cssc.2017.01469.

(41) Che, Q.; Yang, M.; Wang, X.; Yang, Q.; Chen, Y.; Chen, X.; Chen, W.; Hu, J.; Zeng, K.; Yang, H.; Chen, H., Preparation of mesoporous ZSM-5 catalysts using green templates and their performance in biomass catalytic pyrolysis. Bioresour. Technol. 2019, 289, 121729. Doi: 10.1016/j.biortech.2019.121729.

(42) Lok, C.M.; Van Doorn, J.; Almansa, G.A., Promoted ZSM-5 catalysts for the production of bio-aromatics, a review. Renew. Sust. Energ. Rev. 2019, 113, 109248. Doi: 10.1016/j.rser.2019.109248.

(43) Kikhtyanin, O.; Kelbichová, V.; Vitvarová, D.; Kubu, M.; Kubicka, D., Aldol condensation of furfural and acetone on zeolites. Catal. Today 2014, 227, 154-162. Doi: 10.1016/j.cattod.2013.10.059.

(44) Dumitriu, E.; Hulea, V.; Fechete, I.; Auroux, A.; Lacaze, J.-F.; Guimon, C., The aldol condensation of lower aldehydes over MFI zeolites with different acidic properties. Micropor. Mesopor. Mater. 2001, 43, 341-359. Doi: 10.1016/S1387-1811(01)00265-7.

(45) Russo, V.; Hrobar, V.; Mäki-Arvela, P.; Eränen, K.; Sandelin, F.; Di Serio, M.; Salmi, T., Kinetics and modelling of levulinic acid esterficiation in batch and continuous reactors. Top. Catal. 2018, 61, 1856-1865. Doi: 10.1007/s11244-018-0998-y. 
(46) Jacobs, P.A.; Von Ballmoos, R., Framework hydroxyl groups of H-ZSM-5 zeolites. J. Phys. Chem. 1982, 86, 3050-3052. Doi: 10.1021/j100212a046.

(47) Díez, V.K.; Apesteguía, C.R.; Di Cosimo J.I., Aldol condensation of citral with acetone on MgO and alkali-promoted MgO catalysts. J. Catal. 2006, 240, 235-244. Doi: 10.1016/j.cat.2006.04.003. 


\section{FOR TABLE OF CONTENTS USE ONLY}

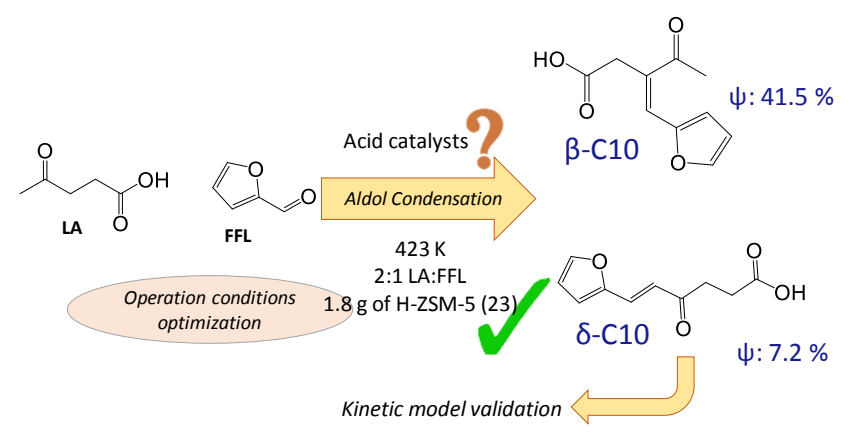

Acid-catalyzed condensation of furfural and levulinic acid is an attractive alternative for obtaining renewable $\mathrm{C} 10$ chemicals at mild conditions. 